\title{
Sculptures transformées, du Phnom Da au Núi Ba Thê
} Bertrand Porte

\section{Citer ce document / Cite this document :}

Porte Bertrand. Sculptures transformées, du Phnom Da au Núi Ba Thê. In: Arts asiatiques, tome 71, 2016. pp. 141-158; doi : https://doi.org/10.3406/arasi.2016.1942 https://www.persee.fr/doc/arasi_0004-3958_2016_num_71_1_1942

Fichier pdf généré le 08/11/2019 


\section{Chroniques}

\section{Sculptures transformées, du Phnom Da au Núi Ba Thê}

«... Nous sommes entrés dans la grotte et nous nous sommes inclinés devant la statue pour lui demander son pardon comme nous l'avions fait pour la statue de Vișnu. Ensuite, nous avons pioché pour dégager la statue de Buddha de son piédestal et nous l'avons emportée hors de la grotte jusqu'au bateau en procession au son bruyant et joyeux des tambours et des gongs. Cela s'est terminé à 5 h 30 du soir, le 6 septembre. Cette statue, auparavant, était en position debout mais elle a été réparée et transformée en position assise à la façon vietnamienne. J'ai trouvé que c'était une bonne idée parce qu'ils avaient conservé tous les morceaux. Les pieds ont été enfouis sous le piédestal pour soutenir les jambes. Les mains ont été fourrées sous les jambes. D'autres morceaux étaient dissimulés dans les deux côtés. Au final, ils avaient réalisé une belle statue avec les bras, l'un sur l'autre. Ils méritent d'être félicités pour avoir bien préservé la statue.»

Kam Doum, « Rapport sur le transfert de statues de Buddha...» (KAM 1945, p. 7). Traduit du khmer par Cham Vicheth.

\footnotetext{
* Remerciements à : Bruno Bruguier, Cham Vicheth, Charlotte Schmid, Chea Sambath, Chea Socheat, Christian Fischer, Christophe Pottier, D.T. Do-Hurinville, Dominique Soutif, Emmanuel Francis, Gabrielle Abbe, Gregory Mikaélian, Jean-Christophe Simon, Marie Aberdam, Marie Christine Uguen, Marie Mellac, Michel Antelme, Pascal Bourdeaux, Pierre Baty, Pierre-Yves Manguin, Preap Chanmara, René Faye, Seila Nhiep, Thierry Zéphir, Y Dary.
}

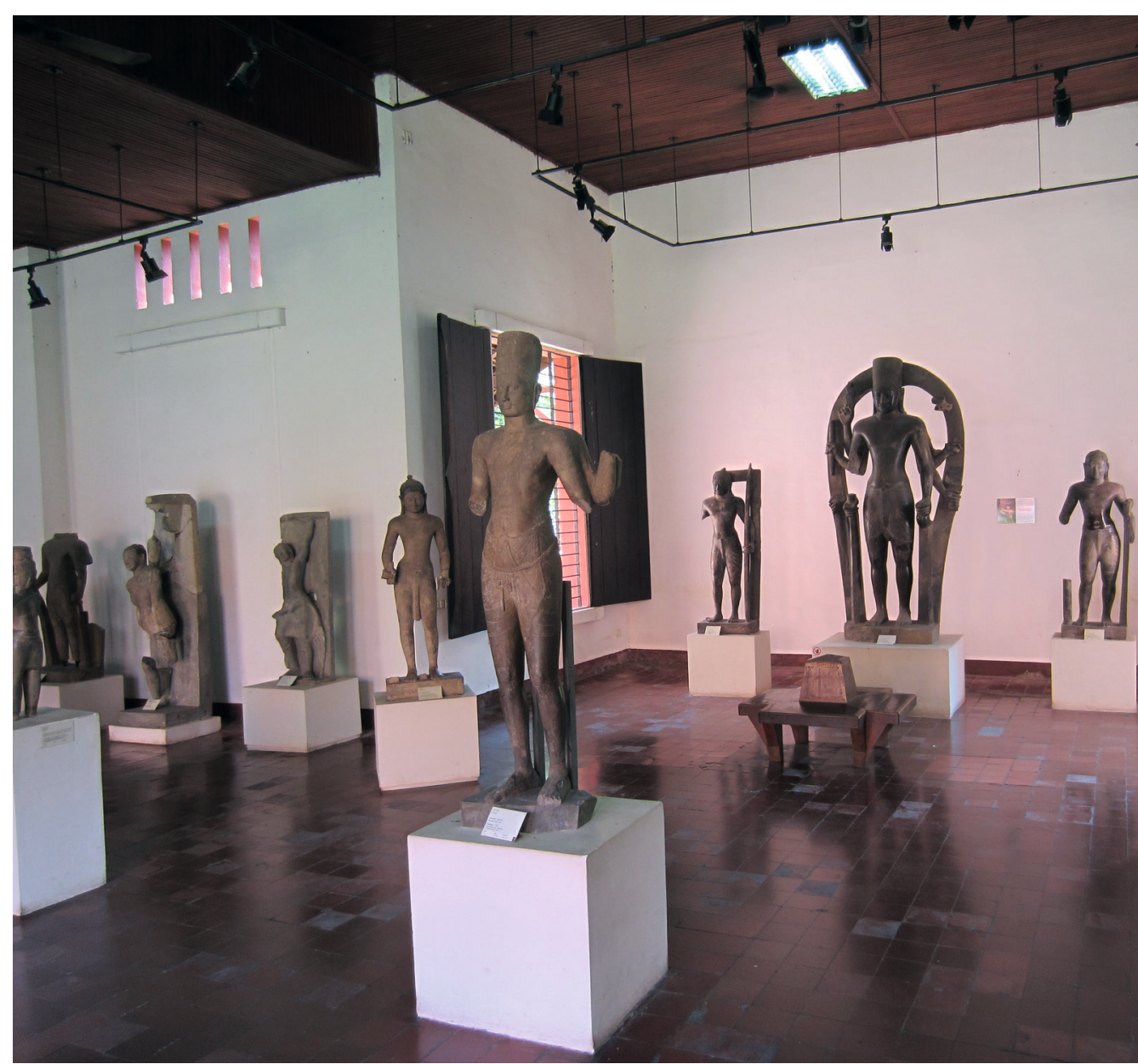

Figure 1. - Le centre de l'aile sud du Musée national du Cambodge en 2014. Au fond le long des murs en partant de la gauche, six sculptures provenant du Phnom Da et une septième découverte à Vat Koh : Harihara ( $k a$ 1614), Krș̣na Govardhana ( $k a$ 1641), Krș̣na Govardhana de Vat Koh (ka 1625), Balarāma (?) (ka 1608), Rāma (ka 1638), Viṣnu à huit bras (ka 1639), Balarāma (ka 1640). Cliché EFEO/MNC. 


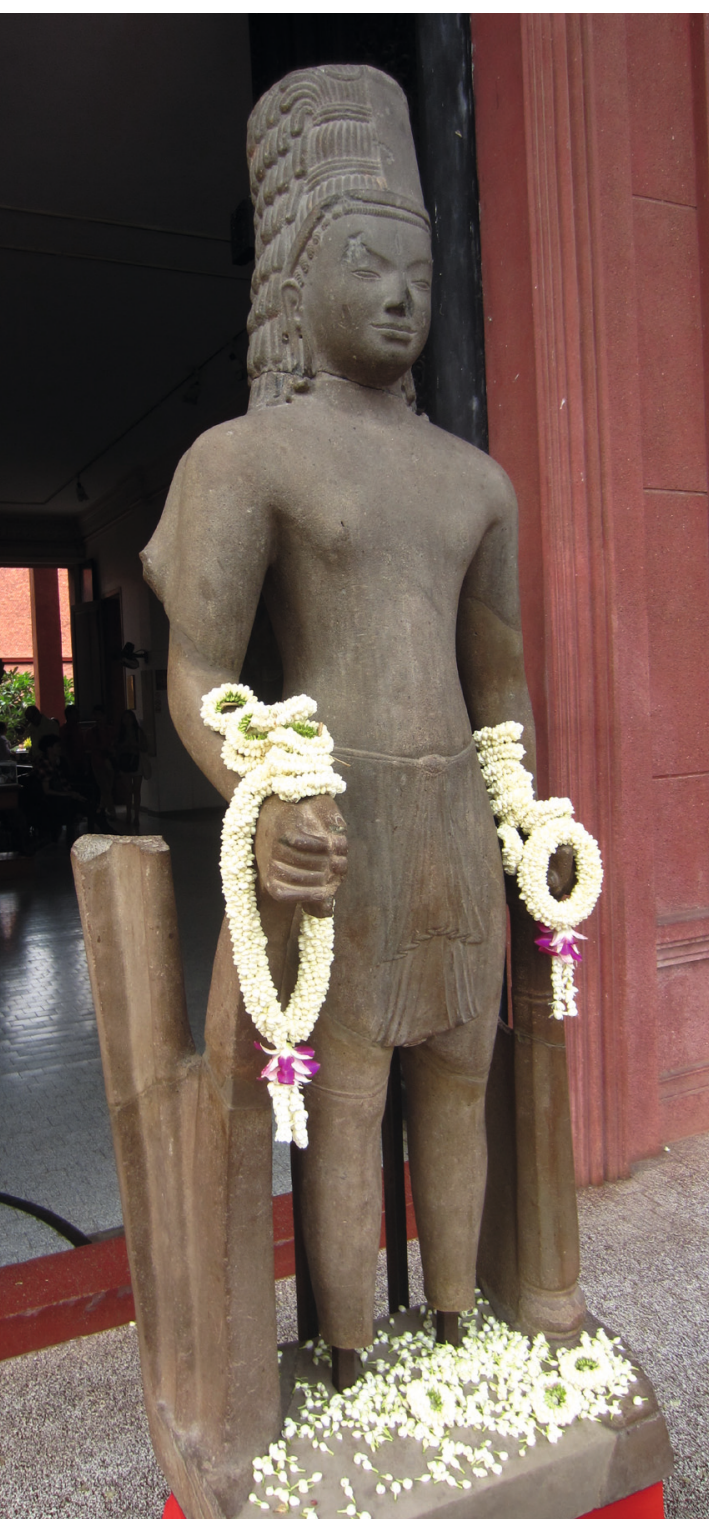

Figure 2. - Le 21 janvier 2016, une tête de Harihara du Phnom Da a retrouvé son corps au MNC grâce à un prêt du musée des arts asiatiques - Guimet.

La tête avait été repérée au Phnom Da et emportée en France par É. Aymonier en 1883 avant d'entrer dans les collections du musée Guimet.

En 1923, G. Groslier recueillit pour le nouveau Musée de Phnom Penh les deux mains inférieures avec leurs attributs, reliés à des portions d'étais. En 1944, P. Dupont et Kam Doum ont rapporté à $\mathrm{Phnom} \mathrm{Penh} \mathrm{le} \mathrm{corps} \mathrm{du} \mathrm{Phnom} \mathrm{Da.}$

En reconstruisant une image de Harihara, auquel il manque encore les pieds, les bras supérieurs et l'arcature de soutien, P. Dupont pressentit alors le rapprochement avec la tête conservée à Paris. En 1998, lors d'une restauration et d'un remontage des onze éléments retrouvés, un moulage du plan de cassure a définitivement confirmé l'appartenance de la tête à cette statue. Cette représentation de Harihara diffère

stylistiquement des autres œuvres du Phnom Da et serait plus tardive. Cliché EFEO.

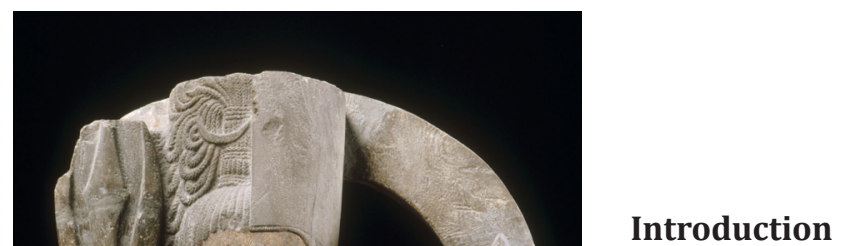

La statuaire du Phnom Da occupe une place majeure dans les collections du Musée national du Cambodge (MNC). Six figures en quasi ronde-bosse ${ }^{1}$ proches de la grandeur nature à l'exception d'un grand Viṣnu à huit bras, y sont entrées par étapes, morcelées, plusieurs fois complétées depuis 1923, jusqu'à très récemment (fig. 1, 2, 3a et 3b). Elles ont toujours été présentées au centre de l'aile sud du musée. Avec deux autres sculptures conservées au musée des arts asiatiques - Guimet et au Cleveland Museum of Art, toutes d'inspiration vishnouites, elles forment, un ensemble exceptionnel ${ }^{2}$, sculpté certainement par les mêmes mains qui auraient œuvré durant la deuxième moitié $\mathrm{du} \mathrm{VI}^{\mathrm{e}}$ siècle sous le règne de Rudravarman, dernier roi du Funan ${ }^{3}$.

À la fin des années 1990, l'état de la présentation des six statues du Musée national du Cambodge était loin d'être satisfaisant. L'atelier de conservationrestauration de sculpture ${ }^{4}$ du musée a mené sur ces dernières des interventions importantes qui se sont échelonnées entre 2000 et 2007 . Elles ont essentiellement consisté à des « dé-restaurations » des précédentes restaurations jugées aujourd'hui, pour la plupart, excessives et plus toujours fiables. Ces travaux suivis de remontages, plus en accord avec les

Figure 3a. - Harihara du Phnom Da emporté en France par É. Aymonier en 1883. Cliché musée des arts asiatiques - Guimet.

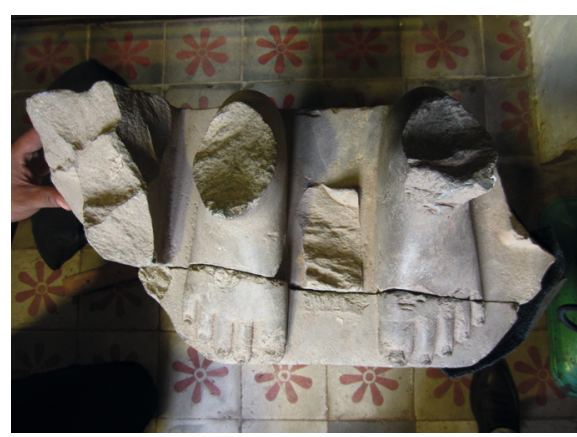

Figure $3 b$. - Les pieds avec base et tenon d'ancrage (cassés en trois) de la même statue rapportés au MNC en septembre 1944 par P. Dupont et Kam Doum. Ils ont été identifiés de nouveau par l'atelier de restauration en septembre 2014. Cliché EFEO/MNC.
1. Il s'agit de sculptures monolithes, quasiment en rondebosse si l'on fait abstraction des étaiements, des arcatures ou des ponts de pierre qui les relient à des chevets.

2. Dans cet ensemble de huit sculptures, seul un Harihara conservé à Phnom Penh (fig. 2) serait un peu plus tardif et appartiendrait à un deuxième style, le style « $\mathrm{B}$ », défini par P. Dupont à partir de la statuaire du Phnom Da (Dupont 1955, p. 42). Voir aussi JEssup \& ZÉPHIR 1997, p. 143-145 ; BAPTISTE \& ZÉPHIR 2008, p. 58-61.

3. Cinq inscriptions lapidaires ont été retrouvées à ce jour au Phnom Da (K. 549, K. 556, K. 670, K. 830 et K. 1216). Trop incomplètes et abîmées elles n'apportent guère d'informations. L'inscription K. 549 sur schiste, bien que plus tardive et difficilement déchiffrable, évoquerait l'ancien roi Rudravarman et l'érection de Hari Kambujendra - Visnu souverain du Cambodge - dans une grotte (COEDÈs 1942, p. 154). Il n'est plus possible aujourd'hui de considérer ces œuvres comme l'émanation du premier art khmer (voir DALSHEIMER \& MANGUIN 1998). Voir aussi BOURDONNEAU 2007 et GOUJON-MARINE 2015.

4. Cet atelier est un partenariat entre l'EFEO et le MNC depuis 1996. 


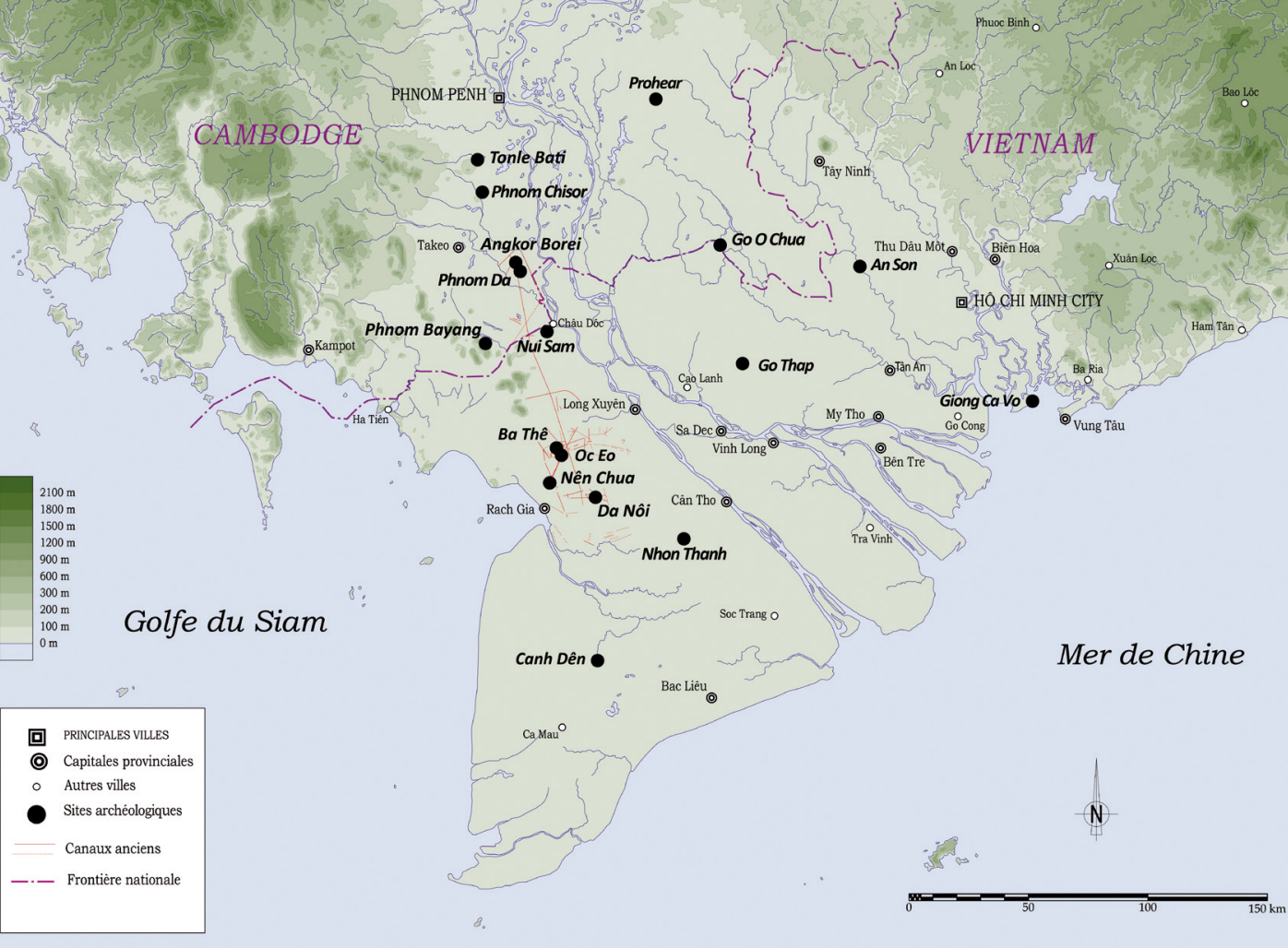

Figure 4a. - Le sud du Cambodge et le delta du Mékong. Carte H. David/P.-Y. Manguin.

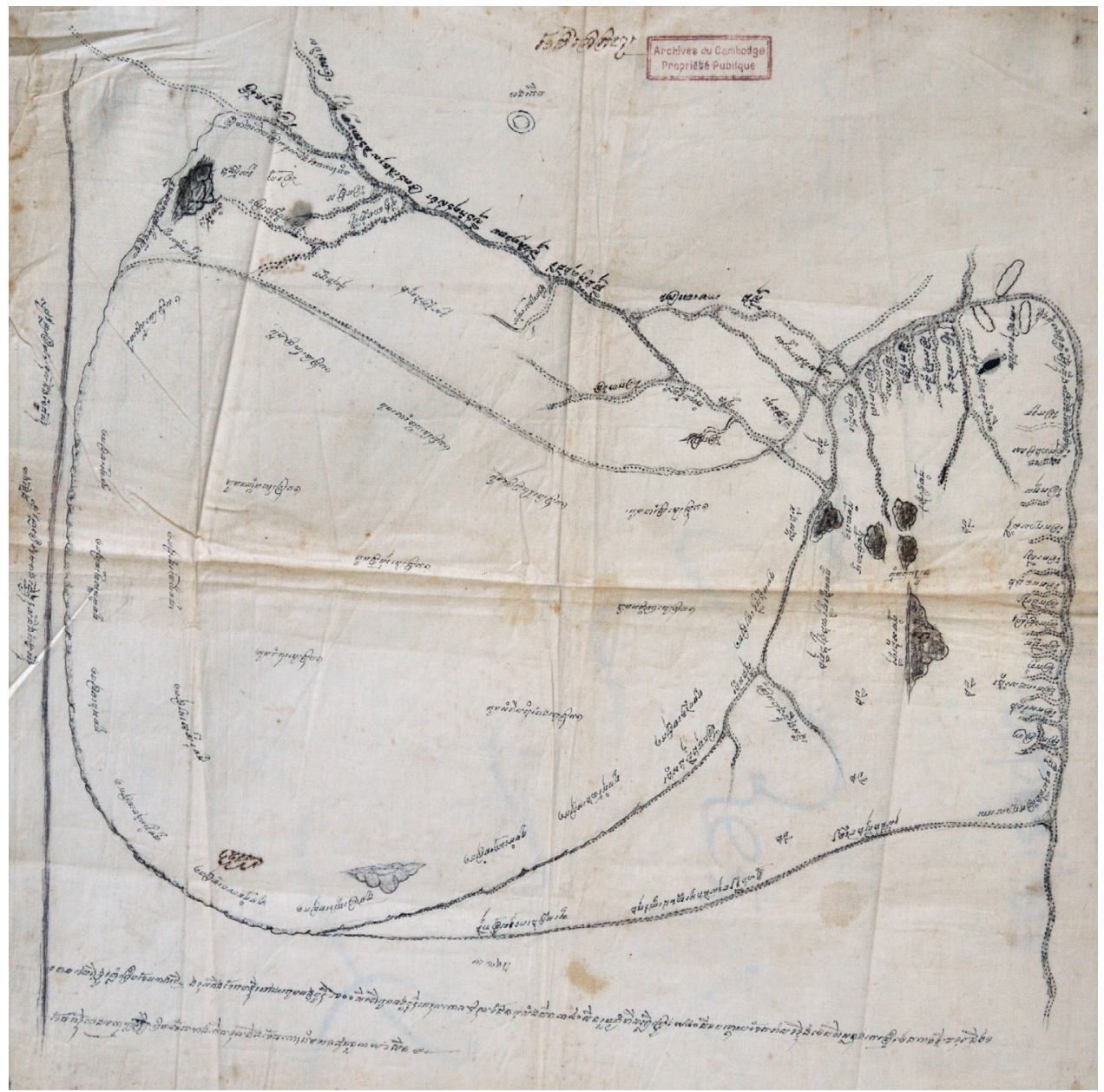

principes de conservation actuels, ont permis de compléter certaines sculptures et de revoir leur mode d'exposition. L'observation attentive des pièces, complétée par de fréquentes visites sur site, la relecture des récits et l'examen des fonds photographiques, ont apporté des éléments explicites. Cette chronique tente de faire le point sur la conservation de ces sculptures et d'apporter précisions et témoignages sur les transformations subies par certaines d'entre elles. Elle nous conduit aussi au Vietnam, plus en aval dans le delta du Mékong, dans le «Transbassac ${ }^{5}$ », pour y observer in situ des sculptures ayant subi des sorts comparables.

Figure 4b. - Carte du district de Pre Krabas, vers 1890. Encre sur toile de coton, $50 \times 50 \mathrm{~cm}$. Archives nationales du Cambodge, réf. ANC 2689/2 Pc2/2.

Le corpus cartographique d'où est tirée cette carte fera l'objet d'une édition intégrale dans le cadre du projet Camnam. La carte est orientée à l'est (indiqué en haut par deux petits cercles). Les tracés et la toponymie changent de sens avec l'orientation, ce qui donne à la représentation l'aspect d'une carte du ciel tournante. Les écritures sont par ailleurs positionnées de façon pourtournante. En bas deux lignes donnent les étendues du territoire dessiné. Les distances sont exprimées en sin (ancienne mesure). Du nord au sud du district de Bati jusqu'à la frontière avec la Cochinchine, il y a environ une trentaine de kilomètres. D'est en ouest le dessin couvre une distance d'environ $12 \mathrm{~km}$. L'échelle diffère selon l'orientation. Les reliefs sont signalés par des circonvolutions en forme de nuages. Au sud un petit groupe de collines (phnom) est contourné par un large méandre de la rivière d'Angkor Borei et de nombreux Prek (bras d'eau). Sont figurés, en partant du sud: le Phnom Borei, le Phnom Kok Loun (aujourd'hui problement le Phnom Ngnil), les Phnom Prasat et Phnom Kiew (les deux autres colines accolées du Phnom Da) et le Phnom Batep vers le nord.

5. À partir de Phnom Penh, le Mékong se subdivise en un bras principal et un bras secondaire. Le Bassac est le bras le plus méridional du delta du Mékong. Au Vietnam il prend le nom de Hâu Giang (litt. Fleuve postérieur), le Mékong prenant le nom de Tiền Giang (litt. Fleuve antérieur). Voir MALLERET 1959, première partie: «L'exploration archéologique du Transbassac ». 


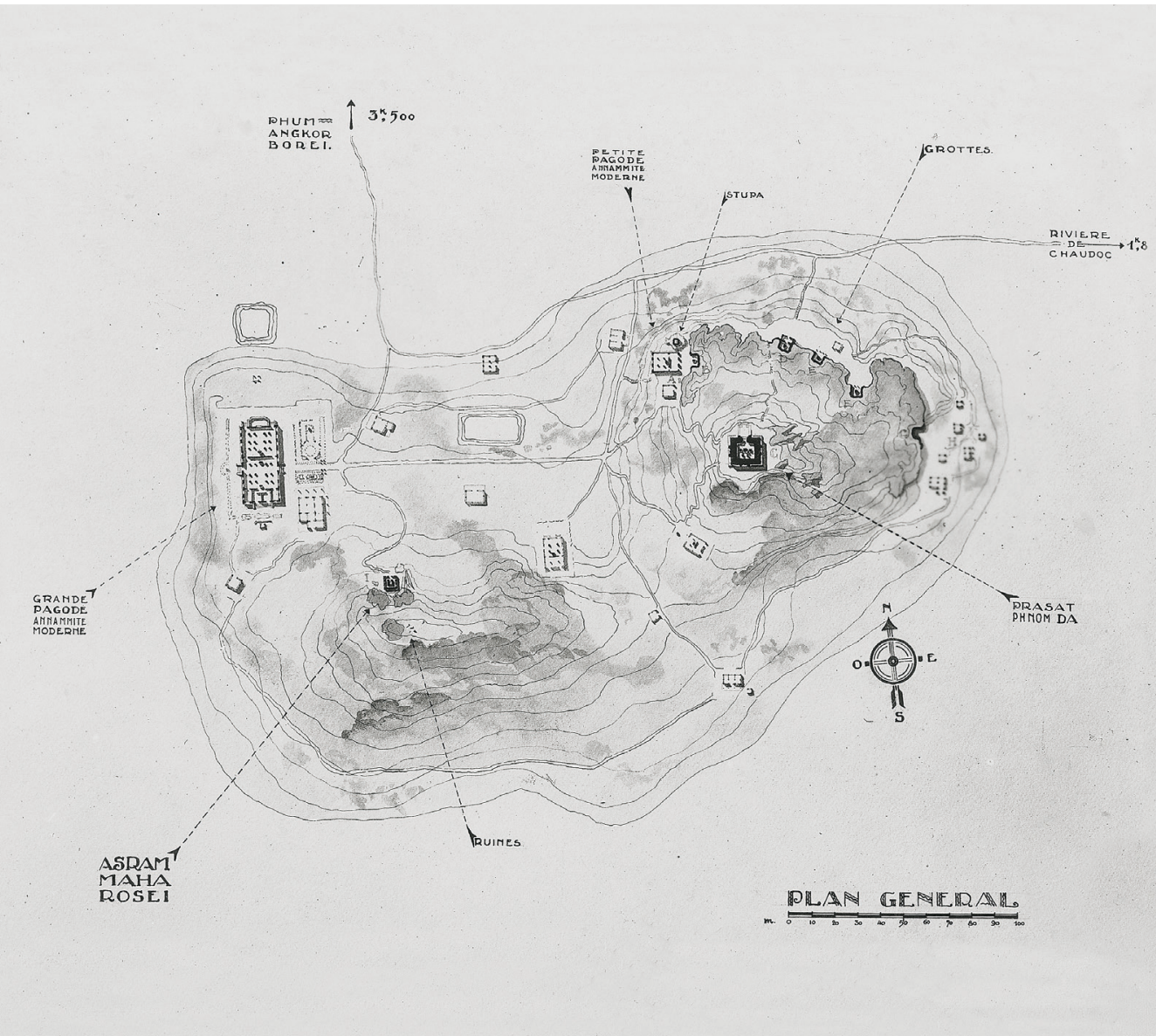

Figure 4c. — Les collines du Phnom Da dessinées par H. Mauger. MAUGER 1936a, p. 66.

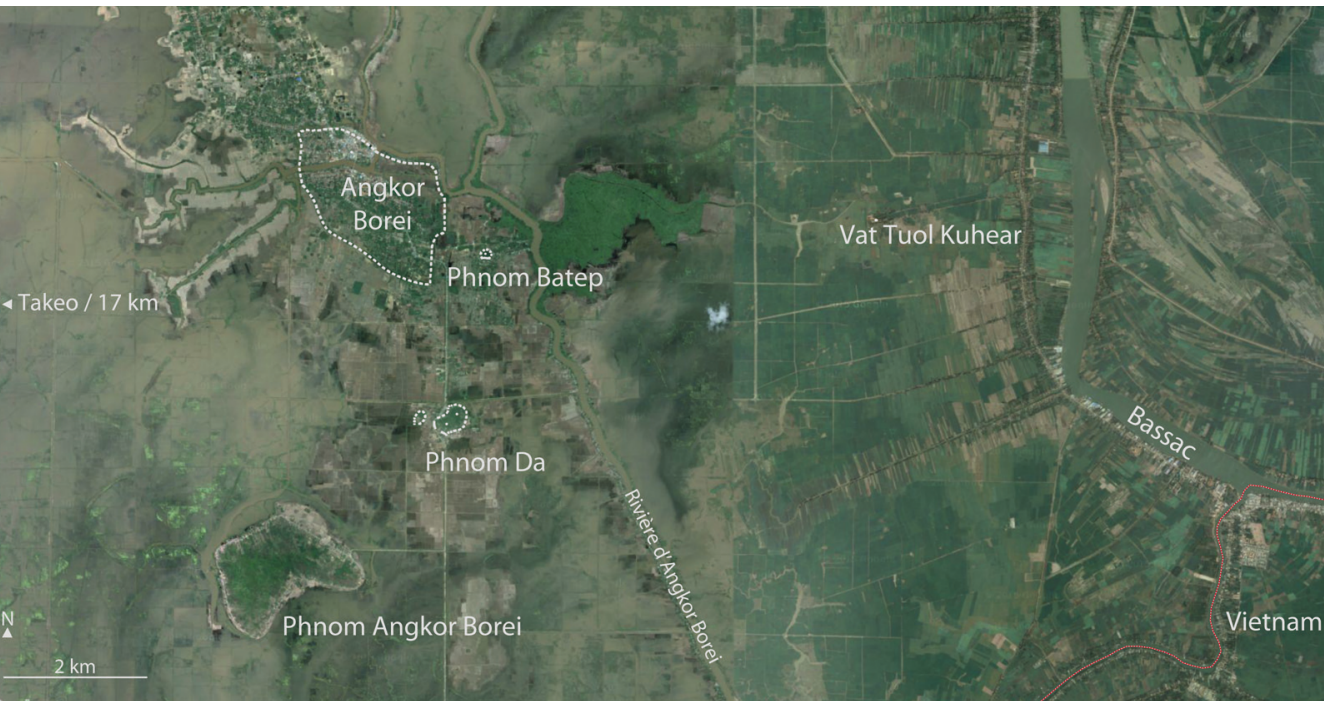

Figure 4d. - Les environs du Phnom Da. La limite entre la province de Takeo (à l'ouest) et le sud de la province de Kandal passe entre le Phnom Batep et Vat Tuol Kuhear. D’après Google Earth.

\section{Le Phnom Da - tour d'horizon des reliefs et vestiges archéologiques ${ }^{6}$}

Plusieurs massifs, collines, ou de simples buttes plus ou moins isolées s'élèvent de part et d'autre de l'actuelle frontière entre le Vietnam et le Cambodge. Derniers sursauts qui parsèment la plaine du Mékong à l'est de la chaîne des Éléphants vers le delta, ils abritent de nombreux sanctuaires établis par les populations indianisées du Funan (fig. 4). Entre ces reliefs, la circulation se fait plus facilement par bateau, en suivant les bras d'eau et canaux ou, parfois, même à travers la plaine, inondée une bonne partie de l'année. On atteint encore aujourd'hui le Phnom Da en embarquant depuis la ville de Takeo, après une demi-heure de navigation plein est en canot hors-bord (fig. 18). On peut également y accéder par le nord en suivant la route récemment élargie.

De nature felsique et grèseuse $\mathrm{e}^{7}$, les reliefs du Phnom $\mathrm{Da}^{8}$ regroupent deux collines jumelles aux pentes rocheuses alignées nord-est/sud-ouest et une troisième plus modeste légèrement détachée à l'ouest (fig. $\mathbf{4 c}$ et $\mathbf{4 d}$ ). Il semble bien qu'au vil ${ }^{\text {e }}$ siècle chacune de ses trois éminences portaient un édifice en brique. La plus septentrionale, le Phnom Prasat (près de $40 \mathrm{~m}$ d'altitude), est aujourd'hui coiffée d'une imposante tour construite en latérite et brique, qui se repère de loin. Cette tour est plus tardive et fut érigée au

\footnotetext{
6. Voir Bruguier \& LaCroix 2009, p. 96-140.

7. Les roches sont constituées d'un grès triasique dans lequel s'est mis en place une rhyolite (équivalent volcanique - ou effusif - d'un granite) qui est probablement associée à l'intrusion granitique du Phnom Borei et aux nombreux reliefs que l'on retrouve dispersés dans la plaine du Mékong (C. Fischer, UCLA/Getty Conservation Program, Cotsen Institute of Archaeology, UCLA). Les statues retrouvées sur le site sont toutes sculptées dans du grès dont l'origine n'est pas connue avec précision. Nous conduisons avec $\mathrm{C}$. Fischer des études sur ces grès afin d'identifier les sources potentielles et d'éventuelles anciennes carrières. Les éléments des piédestaux sont plutôt en schiste ardoisier. Voir Douglas, Carò \& FISCHER 2008

8. Littéralement, "colline de pierre» en khmer E. Aymonier note que l'ensemble des petites collines se dénomme «Nui Long Ton» en vietnamien (AYMonier 1900, p. 199). Il semble bien que cette transcription soit
} inexacte. 
$\mathrm{XI}^{\mathrm{e}}$ siècle à l'emplacement d'un sanctuaire préangkorien ${ }^{9}$. En contre-bas, cinq salles, souvent dénommées grottes ou abris sous roche sont creusées dans les flancs nord ${ }^{10}$. C'est à l'intérieur de ces grottes ou à leurs abords que furent récupérés de nombreux éléments de sculptures.

La deuxième colline, le Phnom Khieu ${ }^{11}$ portait à son sommet une tour sanctuaire en brique extrêmement ruinée et pillée dont il ne reste aujourd'hui que quelques vestiges en élévation noyés dans les broussailles. Sur le versant ouest, à mi-pente, blotti dans les rochers et la végétation, un petit sanctuaire construit essentiellement en blocs de basalte ${ }^{12}$, l'Asram Maha Rosei, est bien conservé. Il a bénéficié d'une anastylose, menée par Henri Mauger en 1936.

La troisième éminence, le Phnom Ngnil $^{13}$, la «colline chauve», un monticule distant d'une centaine de mètres à l'ouest est rarement signalé. Il porte bien son nom car il est bombé et le rocher y est nu sur plus de la moitié de sa surface. Près de son sommet, un édifice totalement arasé ne forme plus qu'une terrasse d'où émergent des briques anciennes. Il semble bien n'avoir jamais été répertorié.

$\overline{9 .}$ H. Mauger eut l'occasion de sonder le mur ouest et retrouva l'ancien parement. Les vestiges de la précédente construction auraient été recouverts de part et d'autre de latérite (MAUGER 1935, p. 490). Une étude du bâti du Prasat $\mathrm{Phnom}$ Da reste à réaliser.

10. Ces salles sont contemporaines des constructions préangkoriennes. Ces cavités de quelques mètres de profondeur abritaient des sanctuaires. L'entrée de la grotte « D » (cotes attribuées par H. Parmentier, deuxième en partant de l'ouest) est encore fermée par une façade appareillée en grès avec une ouverture centrale. Initialement, une avant-structure construite avec d'épais murs de brique devançait la grotte et un encadrement de porte en schiste est resté debout jusqu'à la fin des années 2000 (cf. PARMENTIER 1927, pl. XLVIII et XLVIII [fig. 7a et 7c]).

11. La « colline verte» en khmer, évoque très vraisemblablement le couvert végétal.

12. Voir infra les travaux de H. Mauger. La thèse défendue par H. Mauger est très vraisemblable; les éléments de ce petit temple ont sans doute été importés de la province de Kompong Cham où des basaltes affleurent et où l'on retrouve à Ang Chei, par exemple, le même type de construction. Des éléments de construction en basalte ont été retrouvés épars ou en réemploi à Tuol Kuhear et au Vat Komnou à Angkor Borei.

13. Cette dénomination des trois phnom est employée aujourd'hui et se retrouve sur l'ancienne carte du district (fig. 4b). H. Mauger nomme Phnom Bakhen le Phnom Khieu et Phnom Bakon le Phnom Ngnil. Il ne représente pas ce dernier sur son dessin (fig. 4c).
Très visible à deux kilomètres en direction du sud-ouest, le Phnom Borei ${ }^{14}$ (164 $\mathrm{m}$ ) dessine son profil beaucoup plus massif et allongé. Ses flancs étaient également occupés à l'époque préangkorienne ${ }^{15}$.

À trois kilomètres au nord du Phnom Da, une avancée verte d'où émergent à présent plusieurs antennes, signale l'ancienne cité d'Angkor Borei ${ }^{16}$. La cité est délimitée sur ses six kilomètres de contours par les restes d'une enceinte en brique, bordée d'une douve extérieure. Plusieurs villages sont maintenant établis parmi les nombreux vestiges.

Légèrement au sud-est de l'enceinte, le Phnom Batep ${ }^{17}$, pointe à peine à travers de grands arbres. C'est une petite éminence assez abrupte, où sont amoncelés de très nombreux blocs de granite, de schiste et de grès. Le sommet a été complètement bouleversé pendant la période Khmer rouge, où y fut installé un bastion ${ }^{18}$. Dans le chaos de blocs, on retrouve de nombreuses traces de constructions préangkoriennes dont une pierre de seuil en schiste de large envergure, encore à demi enfouie dans la dépression centrale. Un piédestal de statue particulièrement remarquable, exposé aujourd'hui au $\mathrm{MNC}^{19} \mathrm{y}$ a été découvert.

En continuant vers l'est, à trois kilomètres en direction du Bassac, le site de Tuol Kuhea (un tertre) a livré en 1983 un exceptionnel taureau de Śiva en bronze argenté. Il est présenté dans la Pagode d'argent à Phnom Penh, où il fait l'objet d'une vénération nouvelle ${ }^{20}$.

Enfin dans le lointain s'aperçoivent par temps clair, le Phnom Chisor au nord, ainsi que le profil conique de Núi Sam en direction du sud-est, au Vietnam.

14. Ce nom, en rapport avec la ville d'Angkor Borei, est difficilement traduisible.

15. Cf. РноN 2004.

16. La plaine est en moyenne à $2 \mathrm{~m}$ au-dessus du niveau de la mer. À Angkor Borei l'élévation varie entre 2 et $10 \mathrm{~m}$ (STARK 2003a, p. 93).

17. Ce nom, en rapport avec « divin», est difficilement traduisible.

18. Information recueillie auprès du voisinage.

19. Piédestal de statue $k a 1747$ entré au MNC en septembre 1944. Voir infra le récit de Kam Doum.

20. Porte \& Chea 2008 et ANG 1997

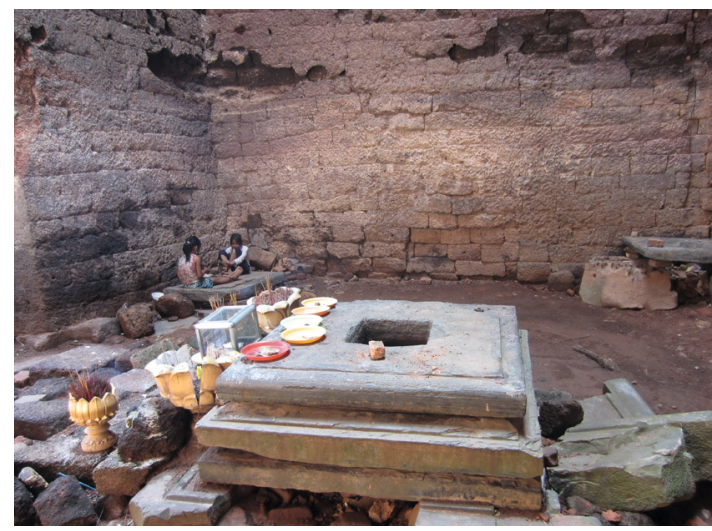

Figure 5. - Éléments de piédestaux en schiste et en grès à l'intérieur du Prasat Phnom Da, septembre 2015. Cliché EFEO/MNC.

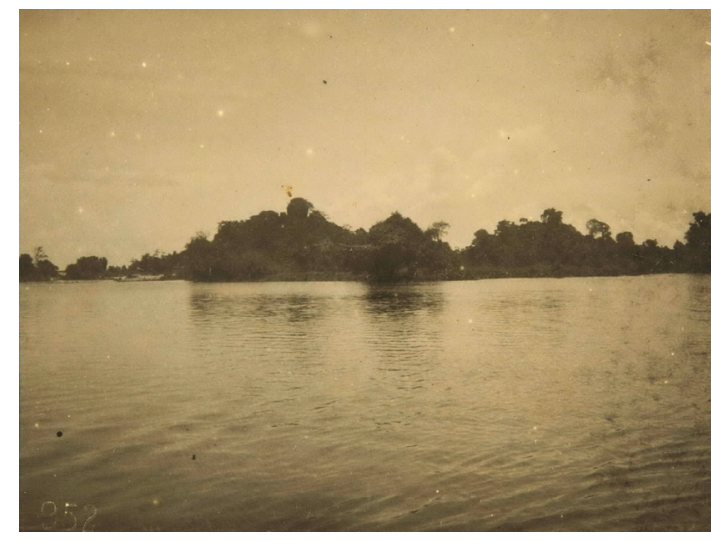

Figure 6a. — Le Phnom Da inondé, ca.1930. Cliché G. Groslier/MNC.

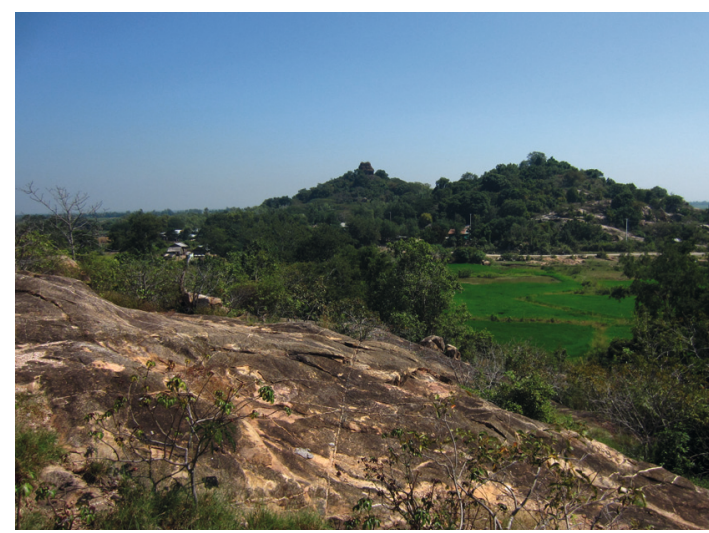

Figure 6b. - Les collines jumelles du Phnom Da, vue depuis le Phnom Ngnil (à l'ouest), janvier 2016. Au premier plan, affleurement de rhyolithe. La photographie est prise depuis une terrasse qui renferme très vraisemblablement les débris d'une construction ancienne totalement arasée. Cliché EFEO/MNC. 


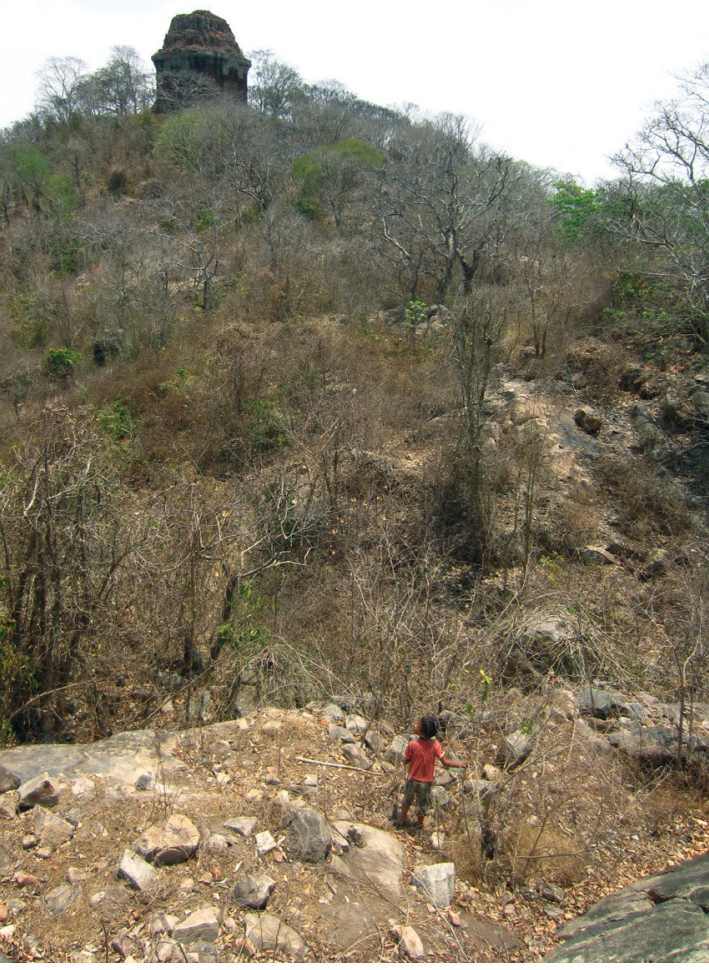

Ci-dessus, de gauche à droite et de haut en bas :

Figure 6c. - Le Prasat Phnom Da en saison sèche, vue du «petit col» qui sépare les deux collines jumelles du Phnom Da, avril 2015.

Cliché EFEO/MNC.

Figures $7 a$. - Plan d'ensemble de la grotte « D » avec l'avant-corps. PARMEN TIER 1927, pl. XLVIII.

Figure $7 b$. - Entrée de la grotte « $\mathrm{D} »$, deuxième en allant de l'ouest vers l'est, $c a$. 1920. L'accroche d'un épais mur en brique est encore visible contre le rocher sur le côté gauche de l'entrée de la grotte (voilé par une empreinte digitale). L'encadrement de porte en schiste de l'avant-corps est encore en place. Cliché EFEO/Fonds Parmentier réf. PARH00315.

Figures $7 c$. - Reconstitution de l'entrée de la grotte « D». PARMEntier 1927, pl. XLIX

Ci-contre

Figure 7d. - La grotte « B», la plus à l'ouest, 2008. Cliché EFEO/MNC.
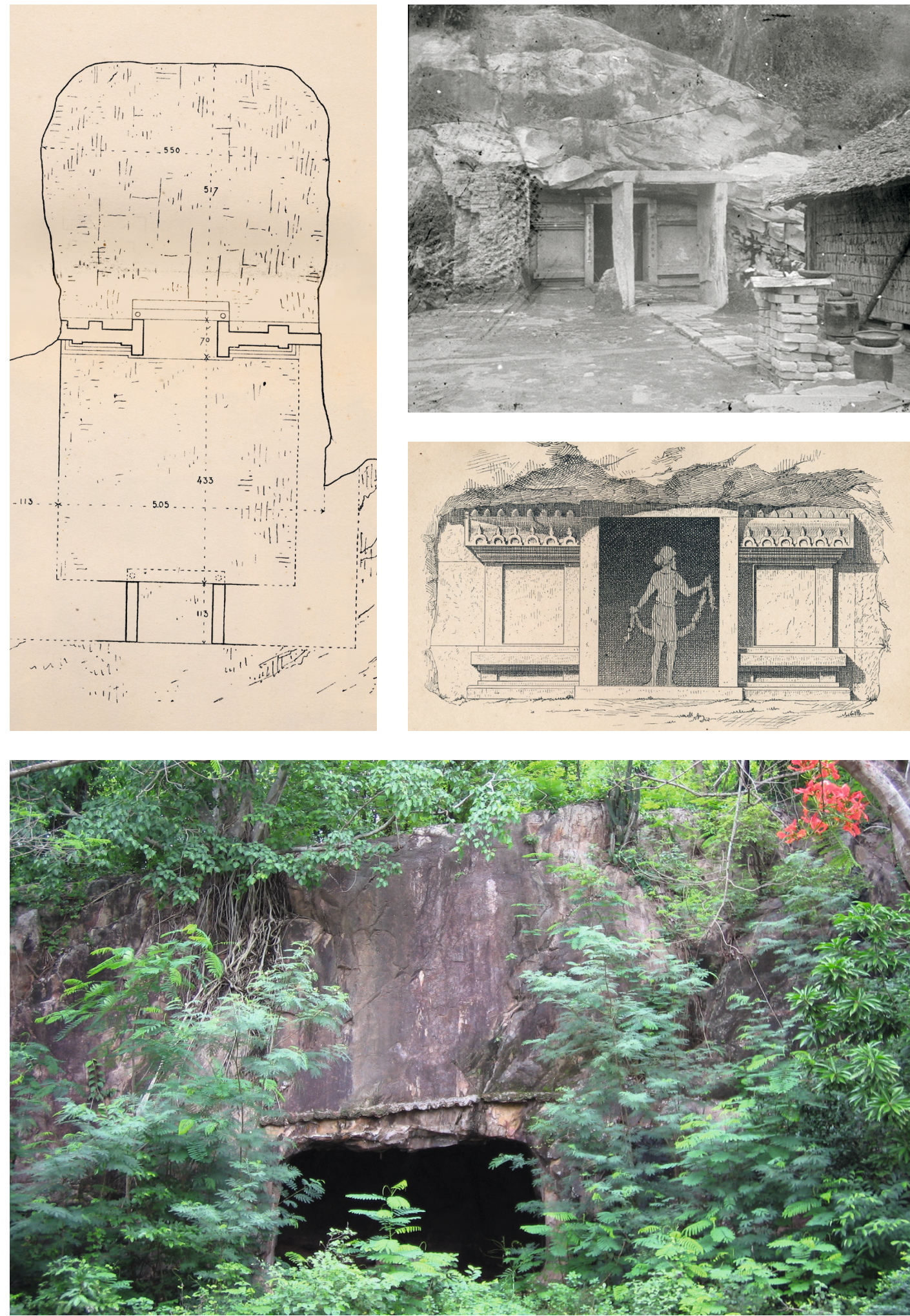

Arts Asiatiques Tome $71-2016$ 
Aujourd'hui la population du Phnom Da se limite à quelques familles cambodgiennes et à une petite pagode, le Vat Phnom Da, établie au pied du flanc nord-est. Au début $\mathrm{du} \mathrm{Xx}^{\mathrm{e}}$ siècle et sans doute jusqu'à la fin des années 1960 des communautés vietnamiennes y résidaient et y avaient édifié des lieux de culte dont il demeure encore quelques traces. Mobiles, ces communautés sont toujours présentes dans la région. $\mathrm{La}$ frontière toute proche et poreuse est propice aux échanges commerciaux qui s'effectuent par bateau. Nous sommes à la croisée des traditions bouddhiques khmères et vietnamiennes, qui a favorisé les réappropriations ou les hybridations de croyances populaires, de symboles et de sites religieux ${ }^{21}$.

\section{Les premiers observateurs et collecteurs}

Les premiers témoignages occidentaux connus relatant la visite du Phnom Da datent de la fin du XIX ${ }^{\mathrm{e}}$ siècle. Louis Delaporte $^{22}$ qui embarqua le 23 juillet 1873 à Saigon pour rejoindre Phnom Penh, après avoir atteint Châu Đốc fit une boucle en remontant la rivière d'Angkor Borei jusqu'au Phnom Da où il nota l'Asram Maha Rosei et les grottes adjacentes.

Chacun à leur tour, Étienne Aymonier (1844-1929), Étienne Lunet de Lajonquière ${ }^{23}$ (1861-1933), George Groslier (1887-1945) et Henri Parmentier ${ }^{24}$ (1871-1949), y passèrent, souvent à plusieurs reprises, et signalèrent la présence de fragments de sculptures dispersés à l'intérieur et à proximité des grottes, devenues pour certaines des lieux de culte vietnamien. Plusieurs pièces importantes furent

21. $C f$. Bourdeaux 2008. Dans le cadre de ses recherches doctorales qui portent sur Thioun, le ministre du palais (1886-1946) et les élites administratives khmères, Marie Aberdam s'intéresse à Son Diep, le ministre de la Marine «khmer Krom », originaire d'une famille de dignitaires vietnamiens, qui avait la charge d'une concession au pied du Phnom Borei où il a financé la construction d'une pagode vers 1920

22. DelapoRte [1880] 1999, p. 26

23. LunET DE LAJONQUiÈre 1902, p. 12-14.

24. PARMentier 1913, p. 4-5. emportées en France ${ }^{25}$. Par la suite, en grande partie grâce aux transferts organisés par H. Mauger et Pierre Dupont, les éléments dispersés (corps, membres, attributs, étais, bases, etc.), de facture souvent remarquable furent transférés au Musée de Phnom Penh alors que d'autres étaient perdus de vue. Les éléments de piédestaux ont été laissés sur place (fig. 5), à l'exception d'une très belle cuve circulaire de linga en schiste dont le canal verseur est creusé dans un bec en forme de tête de buffle ${ }^{26}$. Parmi les fragments sculptés qui attirent l'attention, il est à plusieurs reprises question d'une « main levée » ou d'une figure au bras levé pouvant appartenir à une représentation de Krșna Govardhana. G. Groslier rend également compte de réemplois de sculptures anciennes pour les cultes pratiqués dans les grottes ${ }^{27}$. En 1923 il rapporta deux mains qui furent les premiers éléments provenant du Phnom Da à entrer dans les collections du MNC. Elles étaient toujours présentées telles quelles, perchées sur leurs étais-attributs en $1936^{28}$ au milieu de nombreuses sculptures et de quelques plantes vertes dans l'aile sud nouvellement investie. L'une, la droite, tenant un rosaire, était soutenue par un long étai incurvé, l'autre, la gauche, était appuyée sur l'extrémité d'une massue.

Peu de photographies ont été prises au Phnom Da au début du siècle dernier. Les quelques clichés réalisés par H. Parmentier sont précieux (fig. 7b), de même que ses dessins (fig. 7a et 7c). Aucune véritable fouille archéologique n'y a été conduite jusqu'à nos jours ${ }^{29}$.

25. É.Aymonier a entre autres expédié en France en 1883 une tête de Harihara (MG 14910) ; voir fig. 2.

26. Cuve transférée en 1936 par H. Mauger au Musée de Phnom Penh (ka 1761).

27. GRosLier 1924.

28. Fonds photographique du MNC, plaque de verre 93/3, 1936.

29. Des études et campagnes de fouilles ont été réalisées à Angkor Borei par Miriam Stark (université d'Hawaï) avec le Lower Mekong Archaeological Project (LOMA) en 1995, 1996 et 2003 (voir STARK 1998, 1999, 2003a, 2003b et 2003c). Bong Sovath a participé à ce programme et a fouillé en 1996 au sud du Vat Kompong Luong de l'autre côté de la rivière d'Angkor Borei (BoNG 2003)

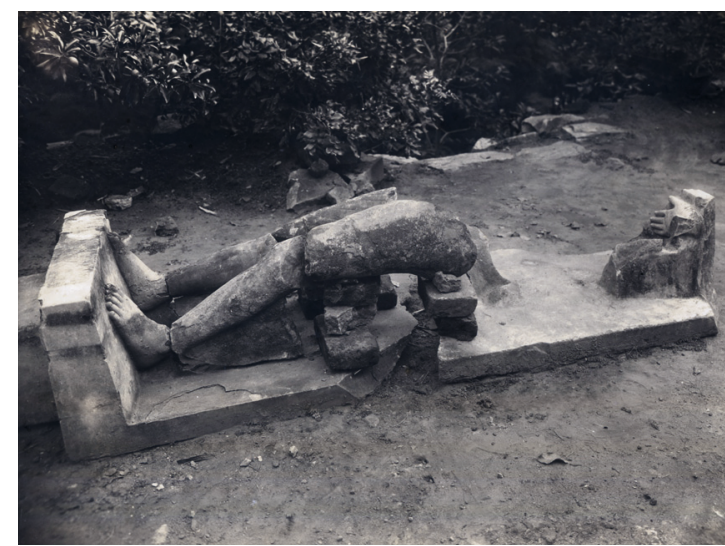

Figure 8. - Restes de l' « homme au bras levé », rassemblés au Phnom Da par H. Mauger, décembre 1935. Cliché EFEO/MNC.

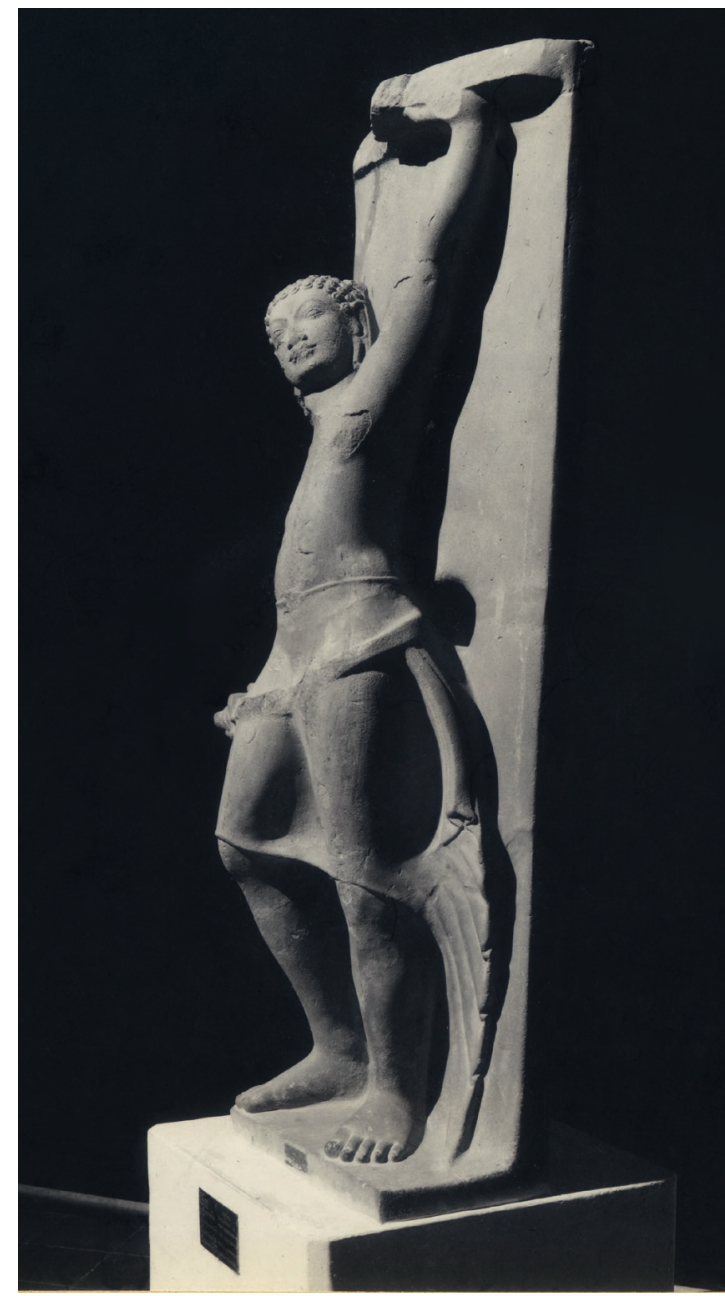

Figure 9. - Kṛșna Govardhana du Vat Koh. Photographié au MNC en 1964. Cliché EFEO/MNC. 

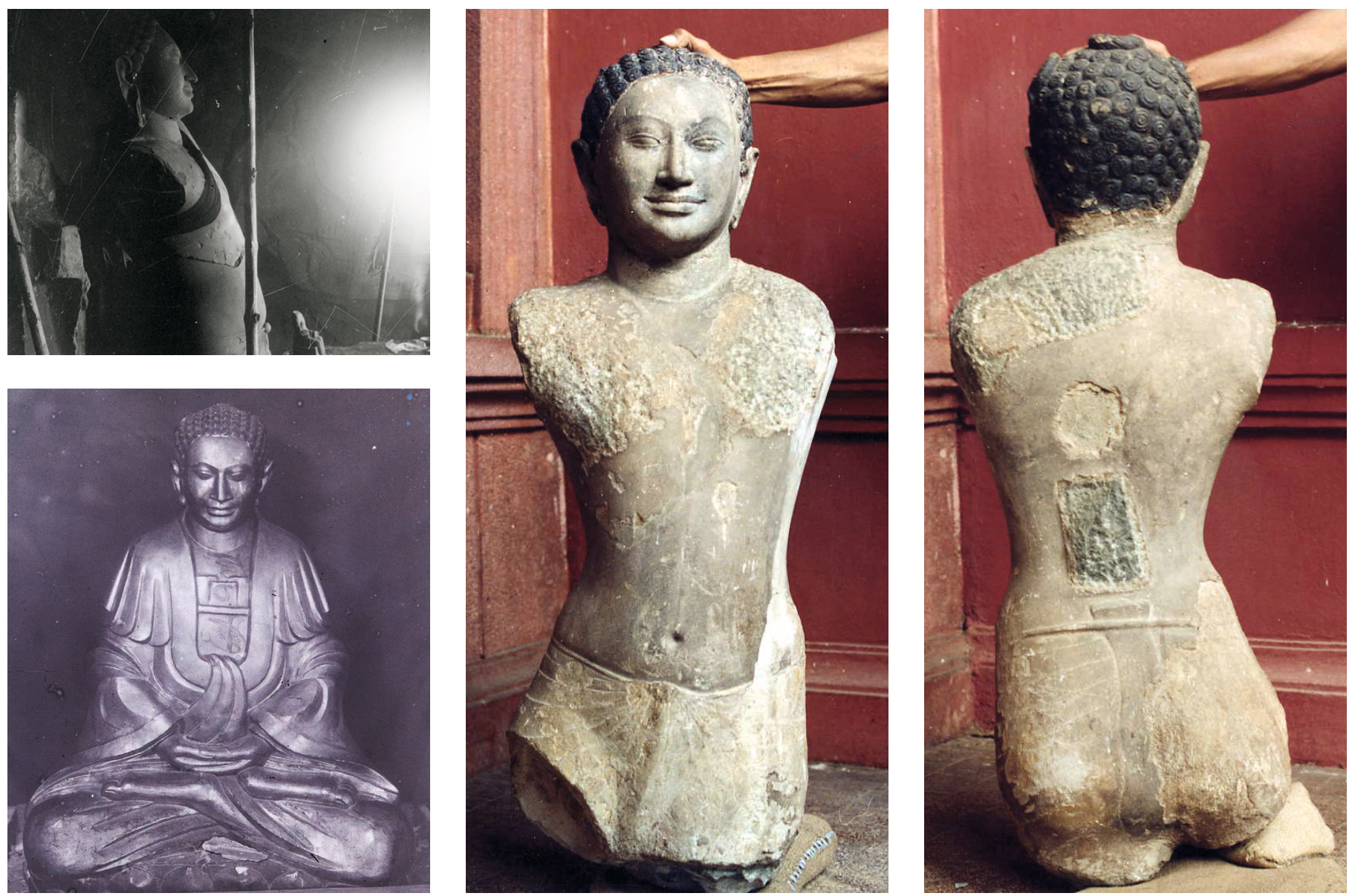

Ci-dessus, de haut en bas et de gauche à droite:

Figure 10. - Buste de Krș̣na Govardhana, photographié dans le contre-jour de l'intérieur d'une grotte du Phnom Da avant sa transformation, $c a$. 1920. On voit un morceau de chevet qui semble correspond re à la statue de Krsna Govardhana aujourd'hui conservée au Cleveland Museum of Art. Cliché EFEO/ Fonds Parmentier, réf. PARH00314.

Figure 11a. - Krsna transformé en Buddha assis, 1944. La photo est vraisemblablement prise au Musée de Phnom Penh au moment de l'arrivée de la statue en septembre 1944. Les signes inscrits sur le plastron n'ont pas été identifiés. Cliché EFEO/MNC

Figures $11 b$ et 11c. - Le torse en 1998 après restauration. Les épaules ont été bûchées ainsi que les deux ponts de pierre arasés au dos. Cliché EFEO/MNC.

\section{Page suivante}

Figures 11d. - Présentation au MNC à partir de 2006 avec des fragments de membres retrouvés. L'avant-bras droit était dans la réserve du MNC. La photo a été prise juste avant que la portion de chevet avec la main gauche levée soit envoyée au Cleveland Museum of Art en octobre 2015. Cliché EFEO/MNC.

\section{Les travaux d'Henri Mauger}

Pendant la saison sèche 1935-1936, H. Mauger (1903-?), directeur de la conservation des monuments historiques du secteur Cochinchine-Cambodge, réalisa l'anastylose de l'Asram Maha Rosei et fit des prospections. Il passa en revue les grottes: «Il y avait là une quantité de fragments - sans intérêt semblait-il - : des tronçons de bras ou de cuisses, des poignets, des doigts et principalement une certaine main que G. Groslier avait déjà remarquée... $\gg^{30}$. C'est ainsi qu'il reconnut des membres, les pieds et la base d'une statue de Krș̣na Govardhana dont le buste se trouvait déjà à Bruxelles dans la collection d'Adolphe Stoclet ${ }^{31}$. Il lui fit envoyer

\section{MAUger 1937, p. 7}

31. Philippe Stoclet raconte que la statue de «l'homme au bras levé » avait été rapportée en France par un marin qui l'aurait échangée contre une moto au marchand d'art parisien Léonce Rosenberg avant que la statue ne fut achetée par A. Stoclet en 1920. ces éléments, ce qui permit d'identifier l'origine de la statue (fig. 8 et 13). À la même époque il découvrit une autre statue de Krșṇa Govardhana en partie prise à l'intérieur d'une termitière dans la pagode de Vat Koh située à douze kilomètres au nord du Phnom Da (fig. 9). Cela portait à deux les statues de Krșṇa Govardhana connues dans le secteur.

Au cours de ces prospections H. Mauger repéra aussi des éléments de statues en contrebas des pentes nord du Prasat Phnom $\mathrm{Da}^{32}$ : une imposante base de sculpture avec pieds et tenon d'ancrage ainsi qu'une main à demi brisée, l'ensemble de taille largement supérieure à la moyenne. Au début de l'année 1936, persuadé qu'une statue correspondant à ces éléments pouvait être cachée sous les décombres qui remplissaient l'intérieur de la tour, il entreprit son

32. Cf. MAUGER 1935, p. 490 et MAUger 1937. La surface des éléments retrouvés à l'extérieur de la tour est beaucoup plus altérée que celle du reste de la statue retrouvée sous les décombres à l'intérieur de la tour. 
déblaiement. Après avoir dégagé une image de Balarāma ( $k a$ 1640), il découvrit la statue attendue, un grand Viṣnu à huit bras ${ }^{33}$ puis une troisième représentant Rāma ${ }^{34}$.

Ces sculptures totalement morcelées furent emportées au MNC pour leur restauration et présentation. H. Mauger ne transféra que les éléments qu'il avait découverts et identifiés ${ }^{35}$. D’autres éléments sculptés restèrentsur place, dont les statues dissimulées par des transformations qui avaient déjà été signalées par certains de ses prédécesseurs.

\section{Le transfert de sculptures sous la direction de Pierre Dupont}

Àla fin de l'année 1944, P. Dupont (19081955), quantà lui, ramassa toutce qu'il put. Il fit transférer un nombre important de sculptures au Musée national qu'il dirigeait alors. Parmi ces sculptures ${ }^{36}$, se trouvaient deux statues du Phnom Da: un buste de Krșna Govardhana (fig. 11b et 11c) et une figure debout à deux bras tenant des amorces d'attributs-étais qu'il n'est pas possible d'identifier précisément ${ }^{37}$. Le buste de Krș̣na Govardhana, antérieurement photographié

33. MNC ka 1639. Désignée et communément reconnue comme «le grand Viṣnu du Phnom Da », l'identification de cette statue n'en demeure pas moins à ce jour incertaine. Les attributs portés, pas tous reconnaissables, ne lui sont pas propres.

34. Le grand Vișnu à huit bras est sculpté dans un grès bien distinct (analyse de C. Fischer). Il est possible qu'initialement seule la statue de Vișnu ait été installée dans la tour au sommet de la colline et que les images de Balarāma et Rāma aient été placées dans les sanctuaires liés aux grottes en contrebas.

35. Les statues de Balarāma, Rāma et Viṣṇu à huit bras ont été totalement de nouveau restaurées ces dernières années. Le pied gauche du grand Vișnu déposé au MNC en 1990 n'a été identifié qu'après.

36. Le registre d'inventaire du MNC enregistre le 25 septembre 1924 (du no 4204 au 4221) l'entrée de 18 objets provenant du Phnom Da. Le ${ }^{\circ} 4205$ signal «trois fragments de pieds avec socle en grès ». Ce sont les pieds (avec base et tenon d'ancrage) de la statue de Harihara retrouvée près de l'Asram Maha Rosei par É. Aymonier, aujourd'hui conservée au musée Guimet. Voir aussi Dupont 1955, p. 25.

37. Jean Boisselier envisage peut-être une représentation de Paraśurāma (BoISSELIER 1955, p. 25, 155 et pl. 6). Aujourd'hui le cartel du MNC désigne Paraśurāma. Il apparaît difficile, dans l'état de la statue, de reconnaître une hache dans ce qu'il reste d'un (des) attribut(s). Il pourrait tout aussi bien s'agir d'une deuxième représentation de Balarāma, le frère aîné de Krș̣na, tenant un araire, étant donné qu'il porte une houppe au sommet de la tête (remarque de C. Schmid). 

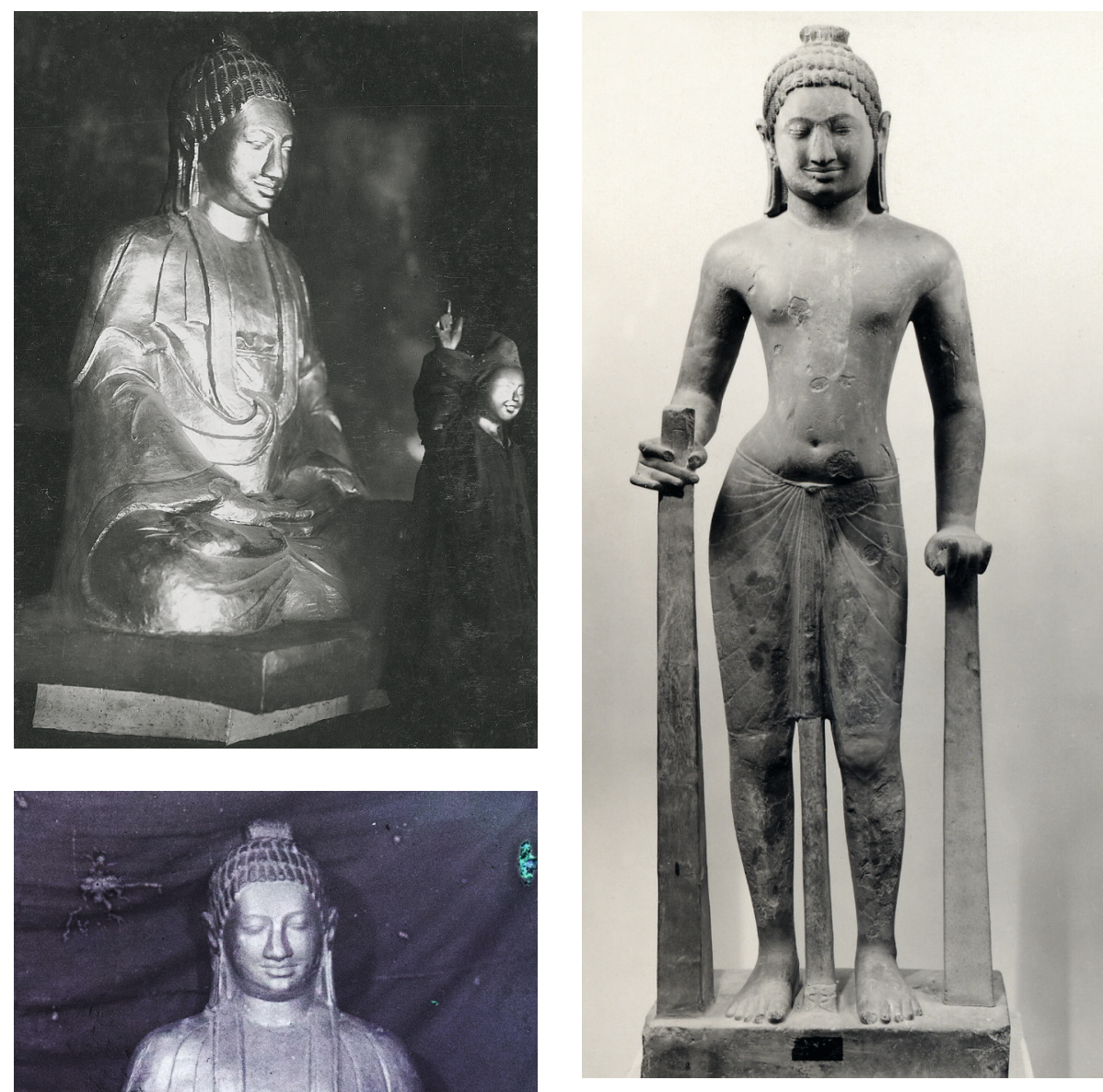

Figure 12c. - La statue restaurée vers 1950 au MNC. Cliché EFEO/MNC.

dans le contre-jour de l'intérieur d'une des grottes par H. Parmentier vers 1920 (fig. 10), n'est plus mentionné ensuite avant son transfert en 1944. Quant à l'autre statue de Krṣṇa du Phnom Da, celle conservée alors à Bruxelles, tout porte à croire qu'on en ignorait l'existence, n'ayant jamais été signalée avant qu'elle ne soit acquise par A. Stoclet en 1924. Plus complète, pourvue de bras et de jambes, son identification ne posa aucune difficulté $^{38}$ (fig. 13).

Ce ne fut pas le cas pour le buste totalement démembré de Krșna Govardhana rapporté par P. Dupont au MNC. Divinité soulevant la montagne, le dieu n'est reconnaissable qu'au mouvement de son torse et de son épaule gauche redressée. Les sections

Ci-dessus, de haut en bas :

Figure 12a. - Statue (Balarāma ?) transformée en Buddha assis à l'intérieur d'une des grottes du Phnom Da, ca. 1930. Cliché EFEO.

Figure 12b. - La même statue extraite de l'autel dans lequel elle était scellée, septembre 1944. Cliché MNC.
38. Une troisième sculpture représentant Krṣna Govardhana a été retrouvée au Vat Koh à $12 \mathrm{~km}$ au nord. Ces sculptures, n'ont pas d'autre comparaison possible qu'avec les deux sculptures Krș̣na Govardhana découvertes sur le site de Si Thep (province de Phetchabun) conservées au Musée national de Bangkok

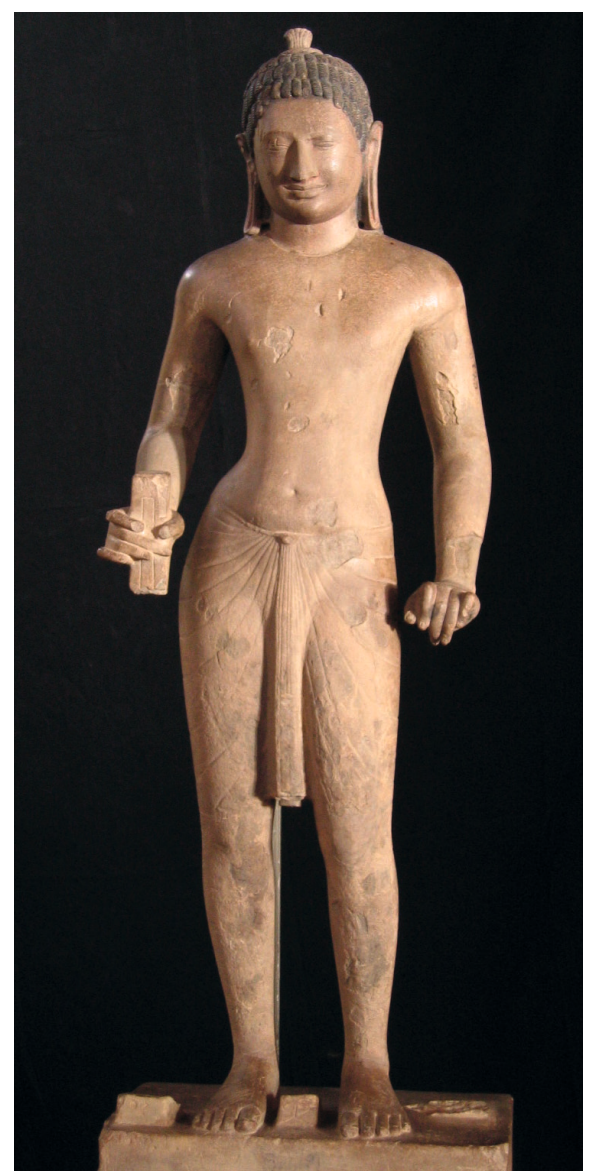

Figure 12d. - La statue en 2007 après une nouvelle restauration. Cliché EFEO/MNC.

d'arrachement des membres et des ponts de pierre, montrent des marques de poinçonnement et de découpes bien apparentes, manifestant une volonté intentionnelle de démembrer et détacher la statue afin de la réemployer. Dans son ouvrage consacré à la statuaire préangkorienne, P. Dupont examine en détail toutes les statues du Phnom Da d'un point de vue iconographique, stylistique et technique. Étonnamment, il ne rend pas du tout compte des conditions et de l'état dans lesquels les deux statues ramenées à Phnom Penh ont été retrouvées et ne mentionne pas les accroches dans le dos du Krșṇa ${ }^{39}$. De la même façon il ne dira rien non plus du Buddha majeur et très vénéré du Vat Kompong Luong à Angkor Borei, qui fut littéralement «enlevé » pour le musée, au même moment ${ }^{40}$.

\section{DuPONT 1955, p. 29}

40. Porte 2002; voir également plus loin le récit de Kam Doum. 
Deux négatifs sur verre restés en marge du fonds photographique du $\mathrm{MNC}^{41}$, qui n’étaient ni inventoriés ni renseignés, montrent deux sculptures de Buddha en posture assise, de facture vietnamienne. On y reconnaît, sous les peintures, les têtes des deux statues rapportées du Phnom Da à la demande de P. Dupont (fig. 11a, 12a et $12 b$ ). La photo est particulièrement explicite pour celle qui avait conservé ses membres inférieurs : on y voit deux fines jambes, dépasser sous une image de Buddha assis en méditation; elles avaient été fichées à l'intérieur d'un autel en maçonnerie qui les dissimulait. Les deux statues ont été remodelées au mortier et transformées en Buddha. Cela rejoint les témoignages de G. Groslier qui mentionnait des images réemployées dans les grottes.

Les deux photos pourraient dater du transfert des statues à Phnom Penh à partir du 25 septembre 1944 (voir infra) et avoir été prises au musée car on peut y reconnaître le motif du carrelage de celui-ci ainsi qu'une grande porte en bois. Avant que l'on détruise les statues pour en extraire les sculptures qu'elles renfermaient, elles ont été moulées en plâtre. Près de dix ans plus tard Madeleine Giteau évoquera très succinctement ce qu'il était advenu de ces statues $^{42}$ et photographia les substituts rendus aux grottes du Phnom Da.

Les copies ont désormais disparu et il n'y a plus de pagode vietnamienne au Phnom Da. Seules demeurent quelques traces matérielles et des témoignages ${ }^{43}$. À l'intérieur des grottes, il ne subsiste que des éléments de piédestaux de la période préangkorienne. Une pagode khmère dont la sala a été récemment agrandie est installée à proximité de la grotte la plus orientale.

Aujourd'hui au MNC, les deux sculptures dégagées conservent des couleurs sur les coiffes et les visages qui témoignent de

41. Plaques identifiées en 2010 lors d'un catalogage. Cote de rangement $87 / 11$ et $87 / 13$.

42. Giteau 1965, p. 41.

43. Une ligne de tuiles appuyées contre la paroi et des décors peints s'observent encore respectivement à l'entrée des deux premières grottes en partant de l'ouest. Un édicule en forme de tourelle est toujours en place entre le Phnom Prasat et le Phnom Khieu. leurs transformations ${ }^{44}$. La statue la plus complète que l'on a envie de reconnaître davantage comme une image de Balarāma que de Paraśurāma (voir note 37) avait été lourdement restaurée lors de son arrivée au musée, avec des reprises d'étais et d'attributs en maçonnerie (fig. 12c). En 2000 la restauration a été complètement reprise par l'atelier de restauration du MNC (EFEO) et allégée (fig. 12d) ${ }^{45}$. Des rajouts en plâtre qui épaississaient le modelé original, intact au-dessous, ont été retirés sur la houppe et la joue droite. Ces remodelages superficiels, forcément récents, avaient surpris au moment de leur retrait. Ils avaient été appliqués lors du réemploi afin de redresser une légère inclinaison de la tête.

\section{Une main levée qui soutient le mont Govardhana}

Les fragments de membres ainsi que les pieds rattachés à une base et tenon et une main levée paume vers le haut reliée à une portion supérieure de chevet, qui furent envoyés à Bruxelles par H. Mauger en 1936 (voir supra) poursuivirent leurs voyages en 1973 jusqu'au Cleveland Museum of Art. Ce n'est qu'à ce moment qu'ils complétèrent la figure de Krșṇa mise sur le marché à la mort d'A. Stoclet et récemment acquise par le Musée de Cleveland (fig. 13). En $2005^{46}$, les restaurations des sculptures du Phnom Da engagées par l'atelier du Musée de Phnom Penh, donnèrent l'occasion au conservateur du Musée de Cleveland d'envoyer à Phnom Penh dix fragments provenant du Phnom Da qui demeuraient non identifiés mais dont tout laissait à penser qu'ils pouvaient compléter les six statues du MNC.

La statue rapportée par P. Dupont qui pourrait être une représentation de Balarāma a ainsi retrouvé son avant-bras droit et son poignet gauche (fig. 12d). Quatre fragments ont permis de compléter

44. Il n'est pas impossible que dans le temps elles aient subit de précédents réemplois.

45. PorTe 2006; voir également le rapport de conservation MNC-EFEO.

46. Cf. Czuma 1974, 1979 et 2005.

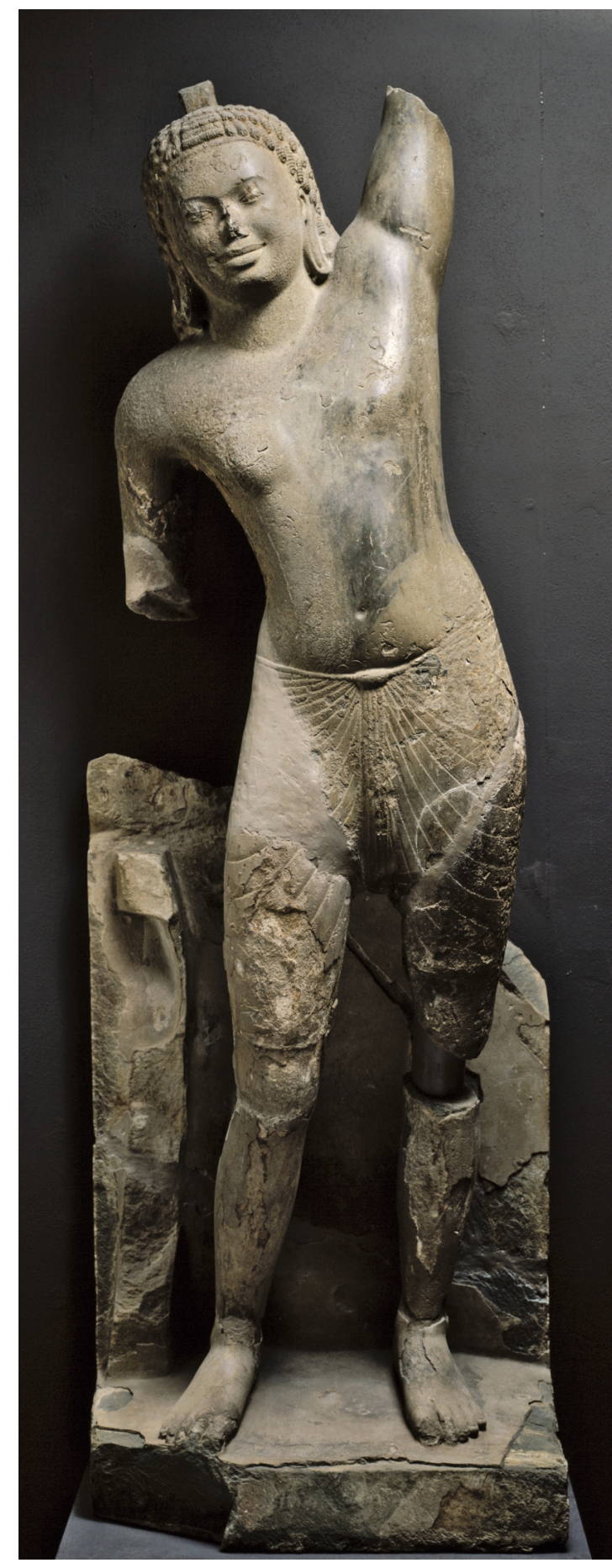

Figure 13. - La statue de Krș̣na Govardhana du Cleveland Museum of Art, qui sera bientôt entièrement démontée puis à nouveau restaurée, afin que soit jointe la partie supérieure du chevet avec la main gauche soulevant le mont Govard hana. 1995. Cliché Cleveland Museum of Art. 
le buste totalement démembré de la statue de Krșṇa Govardhana ${ }^{47}$. Un cinquième fragment, la main levée paume vers le haut reliée à une portion supérieure de chevet, dont l'appartenance au Krș̣na Govardhana de Cleveland n'avait pas été reconnue et dont le rattachement au buste de Phnom Penh paraissait donc naturel, doit leur être ajouté. Les deux Krșna Govardhana de Phnom Penh et de Cleveland sont sculptés en quasi ronde-bosse dans un même grès, même s'ils sont chacun issus de blocs distincts. Un chevet laissé en réserve, partant de la base s'élève en fond jusqu'audessus de la main levée et maintient les figures sculptées par le biais de ponts de pierre reliés aux jambes, aux dos et bras. De proportions assez concordantes avec le Krș̣na Govardhana de Phnom Penh, la main levée paume vers le haut envoyée par le Musée de Cleveland a alors été « greffée par défaut» au dos du buste de Phnom Penh où le positionnement et les arrachements de la pierre de part et d'autre semblent pouvoir correspondre. L'image reconstituée à la demande de la direction du MNC donne une vision plus compréhensible et plus avantageuse du jeune bouvier soulevant la montagne. Des incertitudes subsistaient car aucun véritable raccordement de surface n'était probant avec le dos de la statue, mais il était difficilement imaginable qu'il puisse exister au Phnom Da une troisième ou même une quatrième image de Krșna Govardhana à laquelle aurait pu correspondre la main levée $^{48}$. Depuis 2006, la statue de Krṣṇa ainsi complétée est présentée au MNC au côté de celle du Vat Koh (fig. 11d). Les cinq éléments sont assemblés avec des systèmes de tenon et mortaise bloqués par des goupilles. Les démontages sont relativement aisés et facilitent le transport de l'œuvre qui a été empruntée pour des

47. Le buste de Krșna Govardhana du MNC avait été restauré dès 1997 afin de reprendre plus finement les assemblages de la poitrine et de la face cassées.

48. Deux statues de Krșna Govardhana proviennent du Phnom Da. Au MNC: $k a$ 1641. Au Cleveland Museum of Art : John L. Severance Fund, 1973.106. Une troisième a été retrouvée au nord du Phnom Da et d'Angkor Borei au Vat Koh (MNC $k a$ 1625), dont la provenance n'est pas précise. expositions importantes en Allemagne, en Suisse et à New York $^{49}$.

La greffe était pourtant fautive. Au début de l'année 2014, des photos datant de 1973 transmises par le Musée de Cleveland ont apporté de nouveaux éléments, créant la surprise. Elles montrent, lors d'un essai de reconstitution, la portion de chevet avec la main levée s'adapter au dos de la statue de Cleveland. Les analyses avaient déjà indiqué que le même grès avait été employé pour les deux sculptures. Regardées plus en détail, elles confirment de surcroît que la granulométrie de la roche, souvent variable au sein d'un même bloc, est la même pour l'arrachement au niveau du pont de pierre du côté de la main levée que pour celui situé à la hauteur des omoplates du Krșna de Cleveland ${ }^{50}$. Un moulage et des modélisations en trois dimensions ont achevé de confirmer la concordance.

D'où vient ce contretemps? Le premier propriétaire de l'œuvre, A. Stoclet, ne voulait sans doute pas s'embarrasser de restaurations complexes et parcellaires. Mis à part le pont de pierre au dos, cet élément placé en hauteur ne se raccorde en effet nulle part ailleurs. Il aurait donc préféré abandonner ces éléments fragmentaires pour privilégier l'unité du buste au bras levé. À Cleveland, la mise à l'écart de ce fragment est probablement due à une suite de malentendus et à l'absence de raccordement entre les éléments de la partie inférieure de l'œuvre. Des erreurs de remontage se répercutent inévitablement sur l'axe du corps et gênent le raccordement de la partie haute de l'œuvre.

«La main levée» a finalement refait le voyage et retrouvé sa sculpture d'origine à Cleveland en octobre 2015. Elle sera prochainement installée après un démontage complet des éléments de la statue afin de rectifier les assemblages et mettre en place un nouveau dispositif de soutien.

49. 2007-2008: exposition à Bonn, Berlin et Zurich « Göttliches erbe Kambodschas ». 2014 : exposition à NewYork, «Lost Kingdoms», The Metropolitan Museum of Art. 50. Analyses comparatives des grès (lames minces et spectrométries) réalisées ces dernières années par C. Fischer sur les éléments du Krșna Govardhana du MNC et analyses réalisées sur le Krș̣na du Phnom Da conservé au Cleveland Museum of Art en 1970 par P. Bank.

\section{Le récit de Kam Doum}

P. Dupont ne fit semble-t-il jamais la moindre référence à l'enlèvement des statues du Phnom Da et d'Angkor Borei qu'il a ordonné à la fin de l'année 1944. Les circonstances de cet important transfert sont connues par un rapport rédigé par Kam Doum (1905-?) ${ }^{51}$ membre de l'Institut bouddhique et personnel du Musée national. Tel que publié en 1945 dans la revue Kampuchea Soriya ${ }^{52}$, Kam Doum raconte comment des statues encore vénérées au sein des pagodes du Phnom Da et d'Angkor Borei sont arrivées au Musée de Phnom Penh. Le récit très détaillé de la mission débute à Takeo $(20 \mathrm{~km}$ à l'ouest du Phnom Da) le 4 septembre 1944, en pleine saison des pluies. Les eaux sont hautes. Kam Doum, ses collaborateurs et une équipe de rameurs s'embarquent de nuit avec du matériel en direction du Phnom Da, distant de 20 km vers l'est, à travers la plaine totalement inondée. Au petit matin, l'eau s'étend à perte de vue laissant apparaître sur l'horizon quelques masses sombres signalant des villages et des profils de buttes. Ils atteignent non sans peine le Phnom Da, à travers les nappes de jacinthes d'eau, en début d'après-midi. Aujourd'hui le trajet se fait en trois quarts d'heure en hors-bord dans un paysage qui n'a point changé. Ils sont attendus à la pagode vietnamienne Chùa Tháp Sơn ${ }^{53}$ où ils doivent parlementer avec les religieux pour procéder à l'enlèvement des sculptures. Kam Doum, partagé entre le désarroi de la communauté vietnamienne à voir partir ces statues et les instructions des autorités de Phnom Penh, fait de son mieux pour convaincre son auditoire que le gouvernement est soucieux de la conservation de ces statues. La communauté réclame quelques jours de

51. Nous n'avons aucune information sur sa mort probablement survenue pendant le régime Khmer rouge. 52. КАM 1945.

53. D'après les transcriptions en khmer de Cham Vicheth et les observations de M. Antelme et D. T. Do-Hurinville i s'agit certainement de cette dénomination en vietnamien qui signifie "pagode de la montagne à la tour », correspondant à l'actuel Phnom Prasat. La pagode est installée près de la première grotte. 
délai pour que les populations alentour (jusqu'à Châu Đốc) puissent venir saluer les statues une dernière fois avant l'enlèvement. Kam Doum, bien que compréhensif, est très ennuyé et ne peut leur accorder ce délai. Le 12 septembre, P. Dupont, les autorités de la Province et celles de Phnom Penh ont prévu une cérémonie à Angkor Borei pour marquer la collecte de toutes les statues destinées au Musée national.

Sans attendre, le matin du 6 septembre, l'équipe procède à la dépose d'une statue de Viṣnu ${ }^{54}$ dans un temple voisin, Chùa ông Đại Vương ${ }^{55}$. L'après-midi, une statue de Buddha est enlevée de la «première grotte». Elle recouvre une statue en pied dont les jambes étaient enfouies dans l'autel $^{56}$ (voir infra et fig. 12a).

Kam Doum remarque que les mains originales de la statue ont été remployées pour construire une base sous les jambes en tailleur du Buddha et sont ainsi bien préservées sous la statue. Le lendemain matin, une autre image du Buddha est enlevée à son tour de la « deuxième grotte ». Elle contient le corps démembré d'une statue de Krșṇa Govardhana (voir infra et fig. 11a-b-c).

Pour chaque statue, une procession et des musiciens accompagnent le déplacement jusqu'au chargement dans le bateau. La mission embarque ensuite vers le Phnom Batep (voir infra). Kam Doum note qu'un temple caodaïste ${ }^{57}$ était encore établi au sommet, peu de temps auparavant. Il fait descendre du sommet à grand risque (il faillit se faire écraser) un imposant et très beau piédestal de

54. Il pourrait s'agir d'une statue de Viṣnu très mutilée du XI ${ }^{\mathrm{e}}$ siècle rapportée au MNC ( $\left.k a 218\right)$ lors de ce transfert. Il pourrait encore s'agir du corps de Harihara ka 1614 qui, sans ses attributs et sa tête (voir fig. 2), peut être confondu avec un corps de Viṣnu.

55. D'après les transcriptions en khmer selon Cham Vicheth, M. Antelme et D. T. Do-Hurinville il s'agit vraisemblablement de cette dénomination en vietnamien que l'on pourrait traduire par «pagode du grand roi ». 56. Kam Doum a dégagé et emporté sept éléments de cette statue qu'il est toujours difficile d'identifier aujourd'hui. Voir citation en en-tête, note 37 et fig. 12a-d. $C f$. rapport de conservation MNC-EFEO de la statue ( $k a$ 1608).

57. Nouveau mouvement religieux apparu en 1926 dans le sud du Vietnam prônant un rapprochement des grandes traditions religieuses universelles ( $c f$. BERNARDINI 1975).
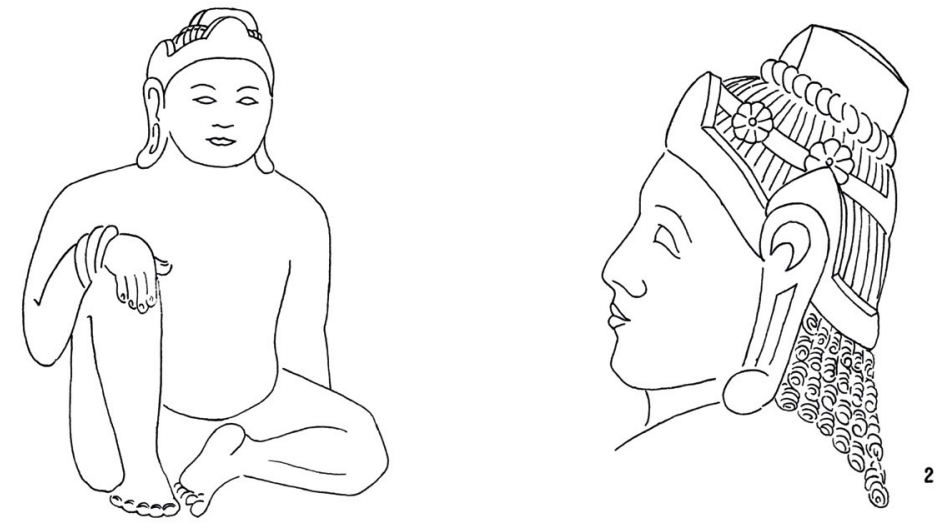

Figure 14. - Dessins de L. Malleret, cherchant à préciser les traits principaux de la divinité de Miếu Bà Chúa Xứ. MALLERET 1943, pl. XVIII.

sculpture. À partir du 11 septembre, la mission est à Angkor Borei où de nombreuses autres pièces sont récupérées - en particulier au Vat Kompong Luong -, dont un remarquable Buddha assis du VI ${ }^{\mathrm{e}}$ siècle encore dissimulé sous de nombreuses restaurations ${ }^{58}$. Des fêtes nautiques et des cérémonies sont organisées aux Vat Kompong Luong et au Vat Chruoy ${ }^{59}$ en l'honneur des divinités. P. Dupont est venu de Phnom Penh avec une délégation. Des présents sont remis aux communautés monastiques, les vénérables sont médaillés ainsi que le chef du village d'Angkor Borei. Une requête solennelle est adressée au vénérable Khy Nut afin d'obtenir son approbation pour l'enlèvement de la statue de Buddha du Vat Kompong Luong. On ressent très nettement, à la lecture du témoignage laissé par Kam Doum, combien la communauté, réticente, contient son mécontentement face aux autorités. Kam Doum et ses collaborateurs durent attendre au-delà de la fête des morts, le 17 septembre, pour parvenir à affréter une embarcation capable d'emporter ce chargement de sculptures volumineux et «sensible». Finalement sous la pression du gouverneur de Takeo, « un Chinois » accepta de prendre en charge le transfert jusqu'à Châu Đốc pour ensuite remonter le Bassac jusqu'à Phnom Penh, où la barge accosta au niveau du marché Sinhek dans

$\overline{\text { 58. Cf. Porte } 2002}$ et GuY 2014, p. 93, Cat 43. Voir aussi le rapport de conservation MNC-EFEO $k a 1731$.

59. Le Vat Chruoy est maintenant désaffecté; son emplacement sur les berges de la rivière d'Angkor Borei était face à l'actuel petit Musée d'Angkor Borei. la nuit du 25 septembre $^{60}$. Le 21 octobre, S. M. Norodom Sihanouk effectue une visite au MNC pour voir les sculptures. Le 23 novembre, le Gouverneur général de l'Indochine accompagné du résident supérieur vient à son tour au musée.

On comprend mieux, avec ce récit, le silence du directeur du musée, P. Dupont. L'empressement que ce dernier mit à faire restaurer et repeindre (trois couches!) la statue du Buddha du Vat Kompong Luong, s'explique quant à lui par l'accident survenu lors de l'arrivée au musée, la statue s'étant brisée en deux (voir note 59).

\section{Les collines de Núi Sam et de Núi Ba Thêê}

Non loin du Phnom Da, au Vietnam, d'autres statues anciennes ont subi des transformations comparables à celles qui viennent d'être retracées. Certaines sont connues, d'autres moins, ou encore même oubliées. Deux sculptures ont spécialement retenu l'attention de Louis Malleret (19011970), lors de ses prospections des massifs

60. Le marché Sinhek n'existe plus aujourd'hui. Son emplacement était quasiment en vis-à-vis du MNC. La physionomie des berges du Tonlé Sap a bien changé depuis. 61. Núi Sam et Núi Ba Thê sont deux termes bien ancrés dans la mentalité populaire locale. Les gens rencontrés ont cependant du mal à en expliquer la signification ou l'origine. L'étude des toponymes de la région méridionale a été engagée en leur temps par Thái Văn Kiêm, Vương Hồng Sển ou Son Nam par exemple. Elle mériterait aujourd'hui une meilleure attention et surtout une plus grande concertation, entre historiens et linguistes notamment, pour confirmer et expliquer selon les cas l'étymologie viet, khmère ou chinoise de ces termes dialectaux. (Communications échangées entre P. Bourdeaux et M. Antelme.) 


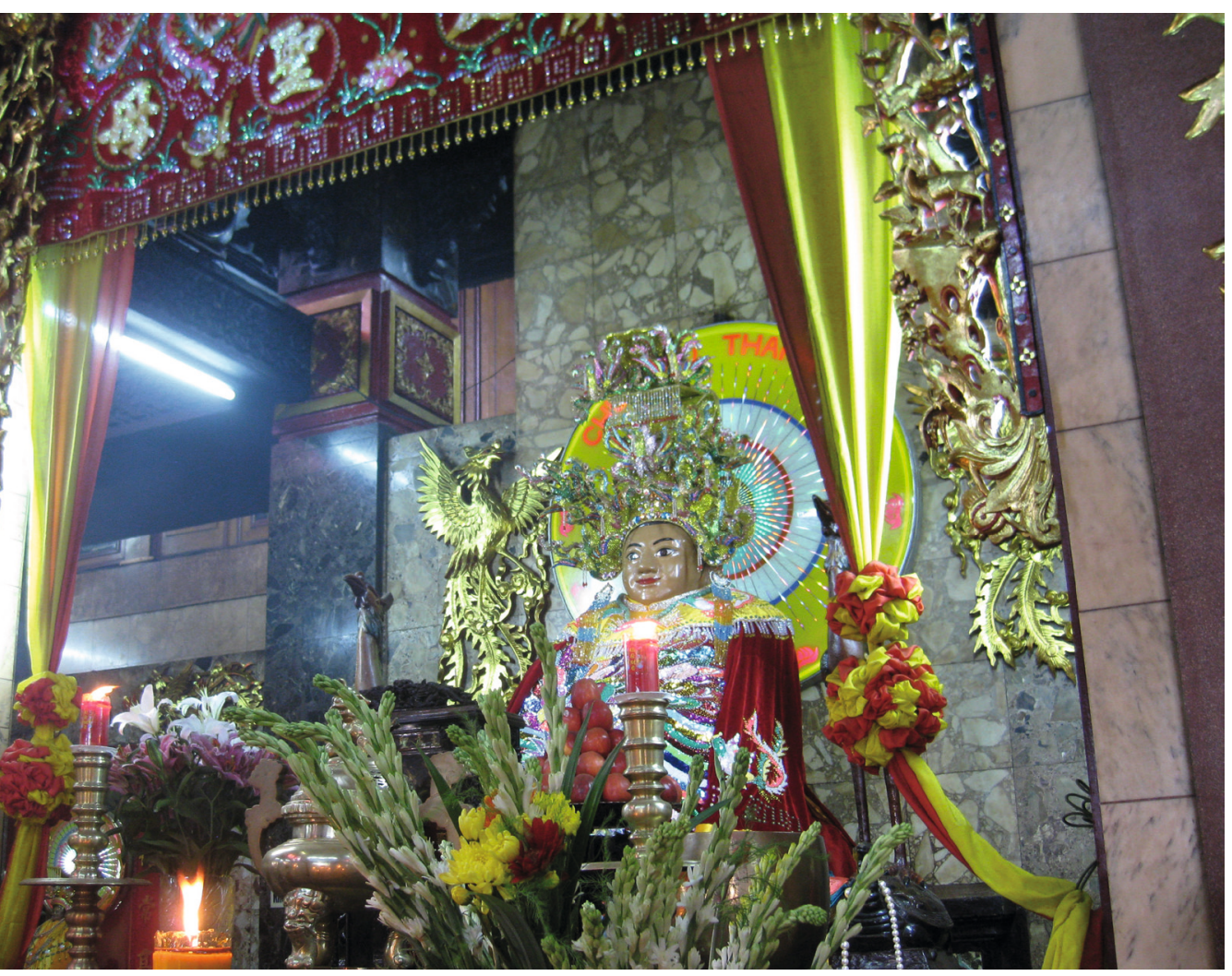

Figure 15. - La divinité de Miếu Bà Chúa Xứ à Núi Sam photographiée en 2013. Cliché EFEO/MNC.

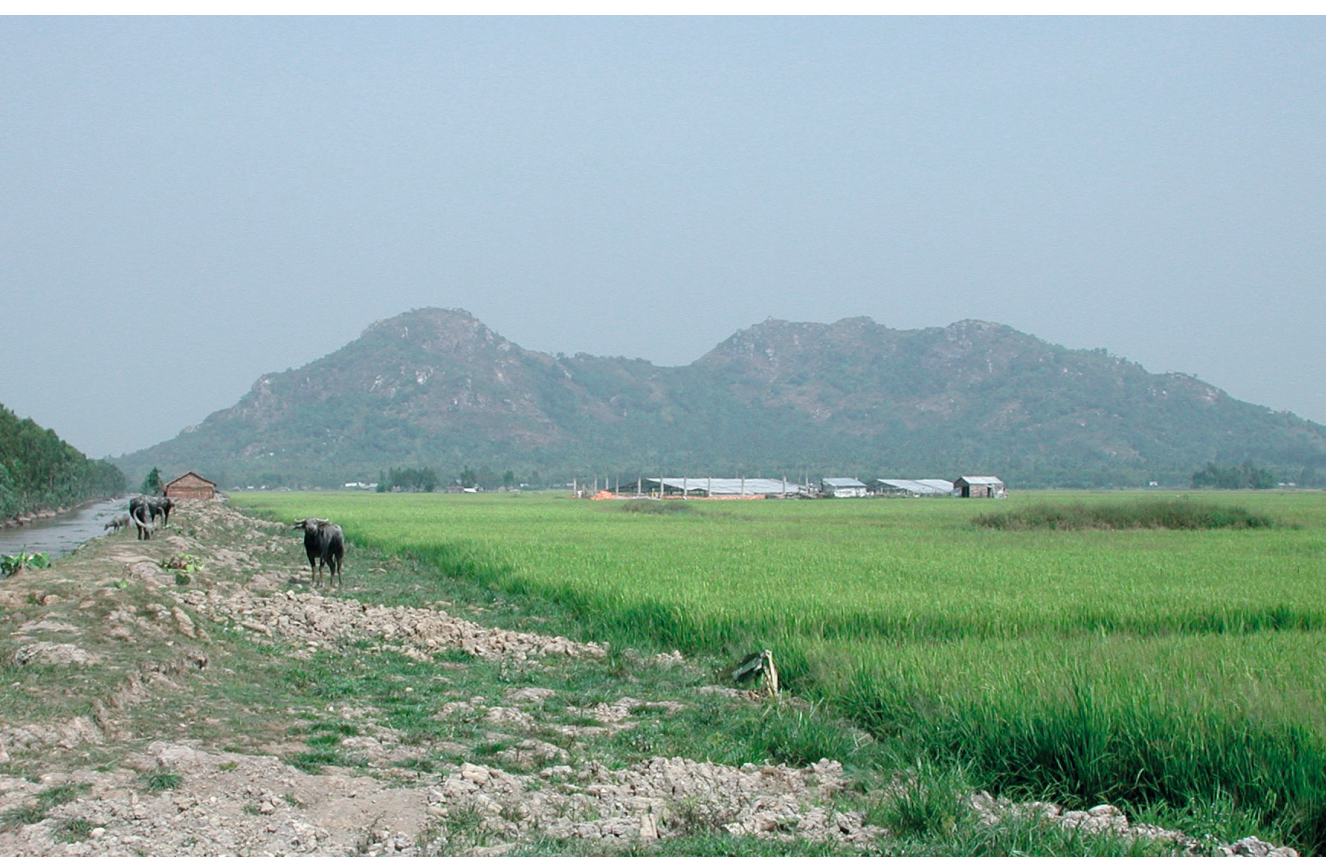

Figure 16. - Núi Ba Thê, vue du sud-est, 2002. Les toitures au ras des rizières signalent un chantier de fouilles sur le site de Go Cay Thy (le «tertre du tamarinier ») dans l'enceinte d'Oc EO. Cliché P.-Y. Manguin. qui jalonnent la plaine du Transbassac entre 1938 et 1945 .

Distante d'une soixantaine de kilomètres au sud-est du Phnom $\mathrm{Da}$, de l'autre côté de la frontière, à $7 \mathrm{~km}$ de la ville de Châu Đốc, le cône isolé de Núi Sam culmine à $230 \mathrm{~m}$. Les alentours sont très riches d'un point de vue archéologique. Au pied nord de la colline, « un petit cirque » densément habité et très commerçant rassemble plusieurs pagodes et un important

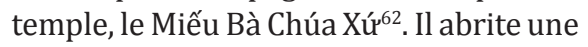
image extrêmement connue dans tout le sud du Vietnam : «Une statue d'un personnage masculin, assis dans l'attitude du délassement royal qui est vénéré par les gens de la région comme divinité féminine et qui a été naturalisé d'une manière bien fâcheuse, en génie vietnamien, par adjonction d'un faux ventre en ciment et de fausses joues enduites à profusion de peintures et de dorure du plus mauvais goût.» L. Malleret poursuit: « Nous avons pu l'étudier non sans peine, après avoir enlevé à la « déesse», huit robes de soie. C'est une idole qui possède des caractères préangkoriens et dut être fort belle... $»^{63}$. Il réussit tout de même à faire une description assez détaillée, en particulier de la tête, et à dessiner ses principaux traits (fig. 14). Par un jeu d'analogies, L. Malleret tend à reconnaître soit une image de Śiva, soit la représentation d'un souverain. Le linga installé au côté de la divinité qui proviendrait, comme la statue, du sommet de la colline, et la lecture par G. Cœdès de l'inscription sur un plateau d'argent (K. 418) trouvée dans une grotte voisine, l'incite à pencher davantage en faveur d'une identification du dieu. Pierre-Yves Manguin, qui réussit à s'approcher de la divinité en 2001, aura beaucoup plus de difficulté à l'observer ${ }^{64}$.

$\overline{62 . ~ L i t t e ́ r a l e m e n t l e ~}$ « temple de la princesse du royaume», consacré à l'un des pèlerinages les plus importants du Vietnam, essentiellement féminin, passant pour procurer la prospérité dans les activités commerciales comme dans la vie privée ( $c f$. TAYLOR 2004).

63. MALLERET 1959, p. 38.

64. P.-Y. Manguin a approché la statue le 4 mars 2001 et note dans son journal : «Visite du site de pèlerinage de Núi Sam. Nous sommes autorisés à passer derrière l'autel de la "déesse du pays" et à soulever sa jupe à l'arrière-gauche. Mains plus grandes que nature (deux fois plus grandes?), 
C'est à peu près tout ce que nous savons de cette image toujours extrêmement vénérée et inaccessible. Elle apparaît en arrière de parterres d'offrandes, baignée dans les fumées d'encens, les lumières clignotantes et le brouhaha des pèlerins ${ }^{65}$ (fig. 15). Aujourd'hui il est interdit de la photographier. Une légende locale raconte que la divinité fut descendue du sommet de la colline. Devenue très lourde quand des Siamois puis des Vietnamiens tentèrent de l'emporter (cent hommes n'y réussirent pas), ce sont finalement des femmes qui la descendirent jusqu'à l'emplacement où elle se trouve maintenant. On pense aux légendes khmères mettant en jeu des rivalités homme-femme qui imprègnent toujours la toponymie ${ }^{66}$.

Plus au sud, au-delà du massif des « sept montagnes » (Thất Sơn), le Núi Ba Thê ${ }^{67}$ domine (à plus de 200 m) l'ancienne cité d'Oc Eo (fig. 16). Le secteur nord-est est particulièrement riche en vestiges de l'époque du Funan et a fourni de nombreuses sculptures déposées au Musée d'histoire d'Hô-Chi-Minh-Ville.

Au début de l'année 1912, une imposante statue en grès ${ }^{68}$ est retrouvée sur une petite butte isolée à l'occasion de la construction d'une maison communale ${ }^{69}$. Brisée au niveau des genoux, elle a quatre bras, le chef mitré et elle est adossée aux cir-

appuyées sur le socle. Aisance royale? Le reste n'est pas reconnaissable.»

65. Le pèlerinage est essentiellement féminin. Jean-Claude Pomonti, qui consacre un beau chapitre au culte de «Dame $\mathrm{Xu}$ » dans son dernier ouvrage, note que ce culte «se traduit, comme beaucoup d'autres, par un mélange d'action de grâce et de requêtes » (PomonTI 2015).

66. SeAng 2001 ; voir également Porte \& Chea 2008. 67. MALLERET 1959, p. 75-96.

68. «Grès fin de couleur gris-bleu, compact et probablement cimenté par de la calcite. La phase argileuse est composée de smectites et d'un peu de chlorite. Âge Triasique. » Lors d'une visite de la pagode de Linh Sơn avec M. Dương Ái Dân, directeur du Musée de la province d'An Giang, des mesures de spectrométrie à fluorescence $\mathrm{X}$ ont été effectuées au revers du capuchon de näga de la statue. Les résultats sont perturbés à cause du fort encrassement mais suffisamment probants pour être comparés au grès d'une autre statue de Vișnu découverte sur le même site, aujourd'hui conservée au Musée d'histoire d'Hô-Chi-Minh-Ville (BTLS 5529). Cf. Bourdeaux, Fischer, Chea \& Porte 2013.

69. Cf. Duvernoy 1924, p. 34-35. Aujourd'hui à l'emplacement du comité populaire de la commune. En 1942 L. Malleret fit des sondages autour de l'ancien đình (maison communale) et fit le relevé des fondations (MALLERET 1959, p. 85, pl. XXXVIII). convolutions d'un serpent dont le chaperon à cinq têtes la nimbe. Quatre ans après sa découverte, H. Parmentier ${ }^{70}$ observe ce qu'il peut de la statue qui a été transportée et convertie en image bouddhique sur l'autel principal la pagode de Linh Sơn, $1200 \mathrm{~m}$ plus au sud-est, au pied du Ba Thề ${ }^{71}$.

À la manière d'une des statues transformées du Phnom Da, les jambes d'origine sont fichées à l'intérieur de l'autel tandis que deux autres jambes croisées sont greffées pour l'asseoir. La statue est en partie remodelée, habillée d'un costume (mantelet) traité de la même façon que celui des statues du Phnom Da. La partie « émergée » mesure près de $2 \mathrm{~m}$ de hauteur ${ }^{72}$. L'autel est flanqué de deux piédroits en schiste, dont un ins$\mathrm{crit}^{73}$, tous deux pris dans les maçonneries.

H. Parmentier en fera un croquis dépouillé faisant abstraction des transformations $^{74}$ (fig. 17a). Il voit une image inhabituelle de Viṣnu endormi sur le serpent Ananta et ne peut déterminer si elle est couchée ou dressée. À la suite des observations de H. Parmentier, L. Malleret consacre un chapitre à l'iconographie de la statue ${ }^{75}$. Entre temps de nouveaux surpeints ont été appliqués, enrobant toujours plus les formes. Il est intéressant de comparer les clichés laissés par H. Parmentier avec l'état actuel de la statue (fig. 17b et 17c). L. Malleret note encore la qualité et la nervosité des traits

\section{PARMENTIER 1923.}

71. MALLERET 1959, p. 80. Le site de Linh Sơn a été fouillé par la mission franco-vietnamienne Archéologie du delta du Mékong en 1998 et 2001. Le tertre central, qui abritait certainement un sanctuaire datant du Funan et de la période préangkorienne, et sur lequel est construite la pagode moderne de Linh Sơn n'a pu être fouillé qu'à sa périphérie. Ces fouilles ont mis au jour au moins deux niveaux de murs d'enceinte entourant ce tertre et, plus loin, des terrasses de bâtiments annexes, avec des occupations couvrant tout le premier millénaire EC (pour un résumé de ces fouilles, voir MANGUIN 2005 et 2009). 72. La statue, d'après L. Malleret, avait jusqu'au niveau des genoux une hauteur de $3,35 \mathrm{~m}$. Nous sommes en présence d'une statue monolithe de taille exceptionnelle. 73. Inscription K. 3 (CoEDÈs 1936, p. 7-9).

74. Dans le dessin la main antérieure droite maintient la boule tournée vers le bas, en pronation, alors que sur les photos de L. Malleret elle est en supination. Peut-être un oubli de $\mathrm{H}$. Parmentier ou bien une restauration de la position de la main?

75. MALLERET 1959, chap. XXIII, «Un problème iconographique : Viṣnu gisant ou Nāga anthropomorphe».

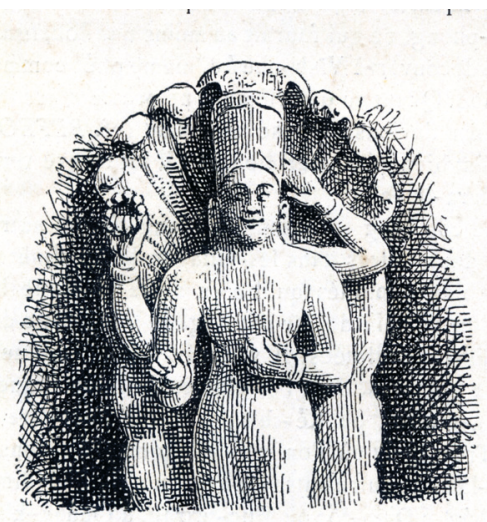

Fig. 1. - Statue de Vıșṿu.

Figure 17a. - Reconstitution dessinée du Viṣnu de la pagode de Linh Sơn. PARMENTIER 1923, p.276.

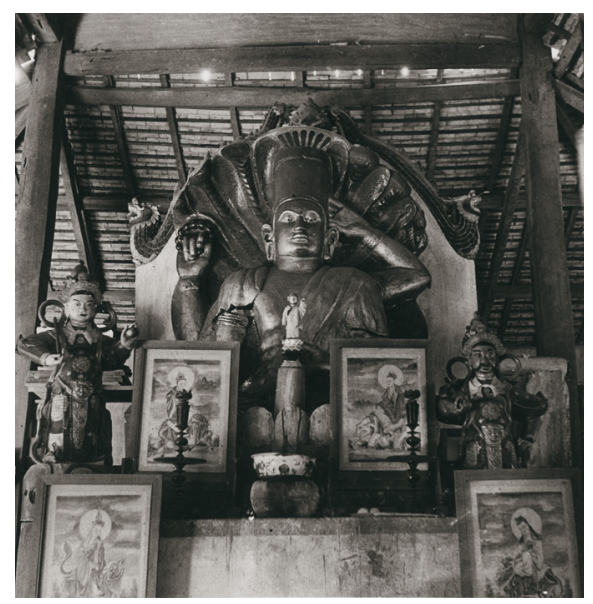

Figure 17b. - Vișṇu de la pagode de Linh Sơn, ca. 1942-1944. Cliché EFEO/L. Malleret.

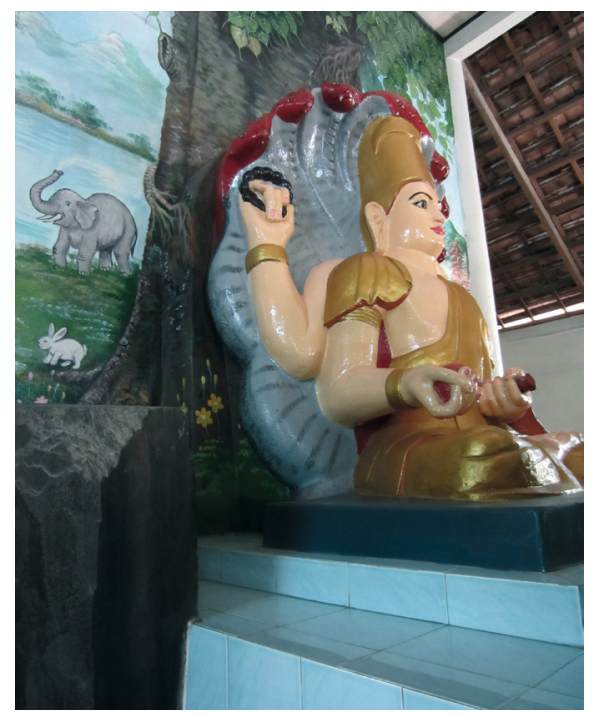

Figure 17c. - Vișṇu de la pagode de Linh Sơn photographié en 2013. Sur le côté, également scellé dans l'autel, un piédroit en schiste porte une inscription (K. 3). Cliché EFEO/MNC. 
au niveau du visage et de la poitrine qu'il est maintenant difficile d'apprécier sous les empâtements brillants.

Ses observations le conduisent à proposer deux interprétations parmi lesquelles il privilégie volontiers la première : un nāga anthropomorphe dressé et un Viṣnu, allongé ou redressé, sur le serpent Ananta. Plutôt qu'une image de Viṣnu telle qu'elle est habituellement représentée, allongée sur le flanc dans des compositions de linteaux, la figuration d'un Viṣnu couché à plat-dos sur le serpent est l'hypothèse aujourd'hui privilégiée ${ }^{76}$. Elle repose sur l'observation de la posture, sa rectitude, la position des bras, la symétrie du capuchon des têtes de serpent, la répartition des enroulements du serpent en quinconce et se satisfait de la comparaison avec les statues que l'on connaît en Inde. Viṣnu regarderait le ciel. Il s'agirait là d'une représentation exceptionnelle par sa taille et son iconographie.

Tout comme pour les sculptures abordées précédemment au Phnom Da et à Núi Sam, nous ne savons pratiquement rien du contexte initial de l'installation de cette sculpture. Elle a été dégagée fortuitement parmi les vestiges d'un sanctuaire installé sur un promontoire détaché des pentes du Núi Ba Thê et formant probablement une île à la période des hautes eaux.

\section{Conclusion}

Le « recyclage» de sculptures au Phnom Da et de celles commentées par H. Parmentier et L. Malleret au Núi Ba Thê sont contemporains, à quelques années près. Dans les deux cas des images funanaises vishnouites ont été réemployées pour être transformées par le bouddhisme vietnamien pratiqué dans le delta du Mékong certainement dès le début du $\mathrm{XIX}^{\mathrm{e}}$ siècle. La transformation de la divinité de Núi Sam, peut-être un peu plus ancienne, relève du même procédé mais pour nourrir, dans ce cas, des croyances

76. Idée proposée par Éric Bourdonneau. locales fortement territorialisées. D'autres exemples similaires sont mentionnés par L. Malleret dans le delta du Mékong et il est probable que des images toujours vénérées aujourd'hui dans la région masquent sous leurs rondeurs et leurs robes des statues anciennes.

Les transformations et réemplois de statues, de fragments de statues et de reliefs pour façonner de nouvelles idoles sont fréquents au Cambodge et au Vietnam de longue date et peuvent revêtir bien des formes différentes. Il est d'ailleurs possible que les statues de Krșṇa et de Balarāma aient déjà fait l'objet de réemplois préalables à ceux du début du $\mathrm{XX}^{\mathrm{e}}$ siècle tels que relatés par Kam Doum. Les restes de dorure sur les visages et la première couche noire sur les cheveux sont peutêtre la trace de réemplois précédents, que seules des analyses pourraient éventuellement confirmer ${ }^{77}$. Dans le même esprit, il est tentant de faire un parallèle avec les fontes rituelles de statues de Buddha toujours pratiquées au Cambodge et attestées dans le passé. Des objets en alliage cuivreux, des parures, de l'or sont inclus en offrande dans la nouvelle fonte. Il s'agit, outre la récupération de l'alliage cuivreux, de nourrir la fonte de métaux précieux ${ }^{78}$. Beaucoup plus récent nous avons l'exemple à Hanoi d'une copie de la statue de la Liberté dénommée Bà đầm xoè (la « Dame robe au vent»), qui fut un temps installée sur le temple de la tortue au milieu du lac Hoàn Kiếm et qui après 1945 fut refondue dans une statue de Buddha à la pagode de Ngu Xà à Hanoi ${ }^{79}$. Les exemples de réemplois effectués pendant les périodes angkoriennes et suivantes ne manquent pas. De très nombreuses sculptures de l'époque du roi Jayavarman VII (fin XII ${ }^{\mathrm{e}}$-début XIII ${ }^{\mathrm{e}}$ siècle) ont été bûchées et re-sculptées par la

77. Rapports de conservation (PoRTE 1996-2016) sur les statues $k a 1641$ et $k a 1608$. Notes sur les restes d'enduits et dorures observés sur les chevelures.

78. VinCENT 2012, p. 330-331.

79. La pagode de Ngu Xà est proche du lac de l'ouest. Cf. DANG 2010. Le film de Nguyen Trinh Thi Lettres de Panduranga (2015), présenté à la galerie du Jeu de Paume à Paris en 2015 fait aussi référence à cette refonte. suite $^{80}$. Les éléments de statues retrouvés à l'intérieur du piédestal du Buddha de Tep Pranam ${ }^{81}$ relèvent davantage du dépôt de fondation tout comme les statues qui peuplaient l'intérieur de la cella de la tour centrale d'Angkor Vat, elle-même murée et transformée en stūpa. Entre amalgames et oublis, il devient difficile de savoir ce que cache une image. Au Vat Nokor de Kompong Cham, par exemple, il est probable que les éléments d'un portrait assis du roi Jayavarman VII, sont noyés dans une représentation en maçonnerie du même monarque ${ }^{82}$. Certains « Neak $\mathrm{Ta}^{83}$ » (génies tutélaires de communauté villageoise) ont pris forme à partir d'une sculpture ancienne. C'est par exemple le cas des jambes et du trône d'un Buddha préangkorien assis pieds au sol, auxquels a été greffé le corps d'un ancêtre local en maçonnerie peinte, qui ont été récemment retrouvés au Vat Tralaeng Kaeng dans la province de Kompong Chhnang ${ }^{84}$. Par extension le jeu des comparaisons peut nous renvoyer vers les sculptures hindoues de l'ancien Champa objets de nouveaux cultes que décrit Paul Mus ${ }^{85}$.

Il est intéressant de remarquer, et pour cela le récit que nous a laissé Kam Doum est précieux, combien il était difficile, malgré les plus hautes autorisations, de venir littéralement arracher une image sacrée et vénérée localement au sein d'un monastère. Ce qui a été possible sous le protectorat du Cambodge dans un silence relatif ne l'a pas été dans le Vietnam colonial ${ }^{86}$. Cela serait encore plus difficile aujourd'hui. La question se pose toujours. Faut-il privilégier la conservation dans un musée ou

\footnotetext{
80. DAGENS 1969, p. 147

81. MARUi 2002, p. 81

82. Communication personnelle de C. Pottier.

83. ANG 1986, FoREST 1992.

84. Cf. Nicolas Revire, infra, p. 159 sq. Le Vat Tralaeng Kaeng est un important monastère installé à l'emplacement du Palais royal de l'ancienne cité de Longvek Des recherches et fouilles archéologiques y sont actuellement dirigées par Martin Polkinghorne avec le concours du ministère de la Culture du Cambodge, du Centre de recherche sur le patrimoine de Nara (Japon) et de l'université de Flinders (Australie).

85. Mus 1933, p. 371

86. Cf. Mauger $1936 \mathrm{~b}$
} 


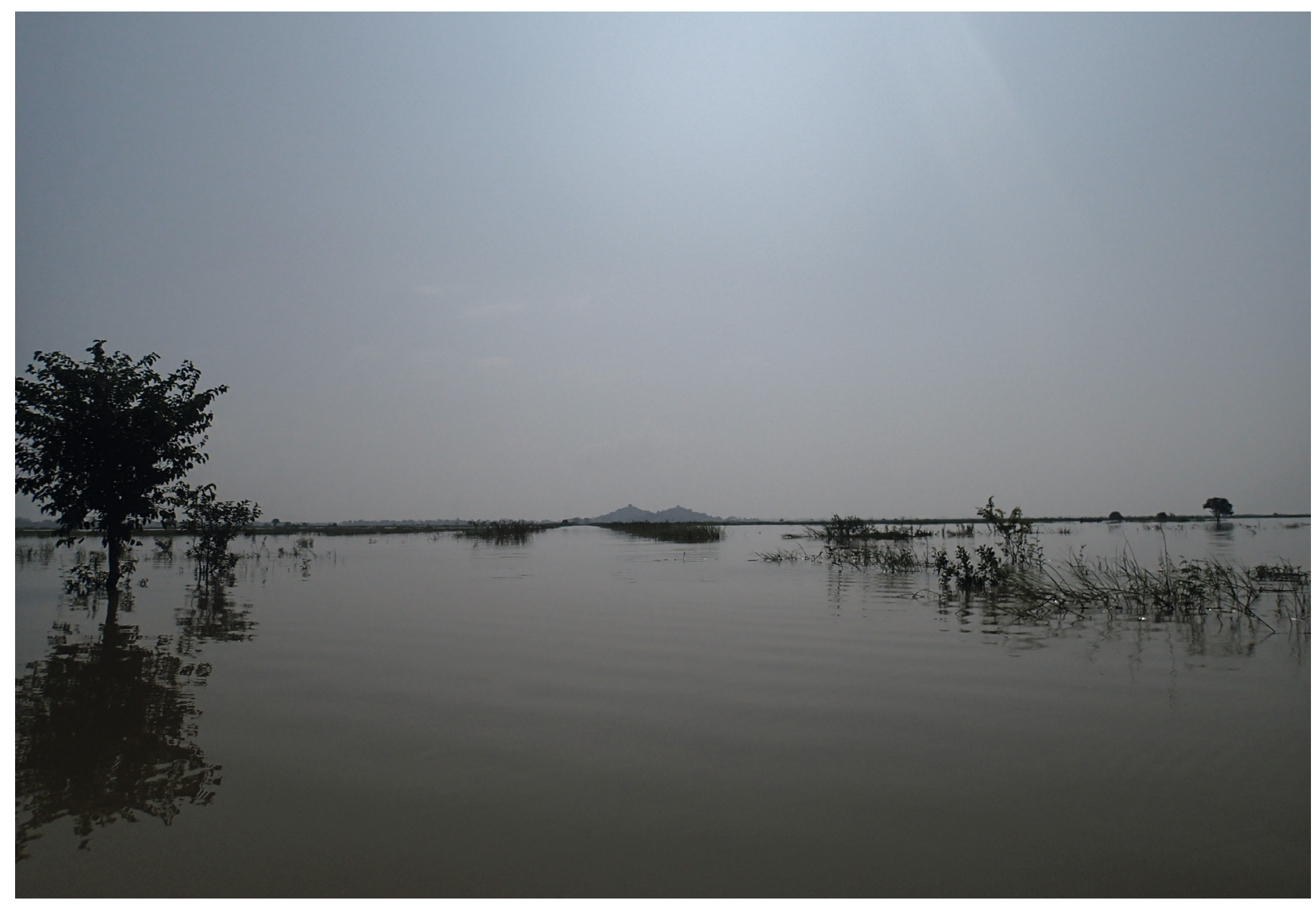

Figure 18. - Les collines du Phnom Da à l'horizon après 10 mn de navigation plein est à partir de la ville de Takeo, septembre 2015. Cliché EFEO/MNC.

la dévotion? En ce sens les remarques de Kam Doum ( $c f$. citation en en-tête) sont pertinentes. En effet dans la mesure où le remploi ne met pas en danger la bonne conservation de l'œuvre originale, son démontage à des fins muséographiques peut paraître contestable car il ne respecte pas les pratiques religieuses. Toutefois des déposes peuvent être justifiées en raison d'incompatibilité avérée des matériaux ou de conditions de conservation inadéquates. L'histoire a finalement donné raison à l'intervention de Kam Doum ; les conflits qui ont éclaté à partir des années 1970 et le régime Khmer rouge n'auraient certainement pas épargné ces œuvres. La pagode et les sanctuaires qui les abritaient ont disparu. Rappelons que l'enlèvement du Buddha de Vat Kompong Luong à Angkor Borei au même moment a permis sa sauvegarde car la pagode fut totalement détruite.

Ce que l'on sait de la disposition originelle de ces statues dont la matière première (le grès) a été importé, est ténu. Les édifices ou les cavités qui les abritaient s'étageaient des sommets aux pieds des escarpements. Des éléments de piédestaux en schiste ou en grès demeurent encore parfois sur place, épars, empilés ou à demi enfouis. Tout juste peut-on imaginer abrités dans de sanctuaires en brique, l'actuelle «Bà Chúa Xứ » trônant au sommet de Núi Sam ou au Núi Ba Thê, le Vișnu de la pagode de Linh Sơn, allongé au pied de la montagne. Au Phnom Da, un grand Vișnu à huit bras dominant la plaine, avec en contrebas, sous le rocher, au-dessus de la ligne des eaux, comme il le fait à Mahābalipuram, Krșna soulevant la colline $\mathrm{e}^{87}$.

Bertrand Porte, EFEO Phnom Penh bertrand.porte@efeo.net

87. C. Schmid, «Pluie et Pierre», table ronde avec É. Bourdonneau et B. Porte, Festival d'histoire de l'art, Fontainebleau, 31 mai 2015 


\section{Bibliographie}

ANG Choulean, 1986 : Les êtres surnaturels dans la religion populaire khmère, Paris, Cedoreck.

- 1997: "Nandin et ses avatars », in Jessup Helen I. \& ZÉPHIR Thierry (dir.), Angkor et dix siècles d'art khmer, Paris, Réunion des musées nationaux, p. 62-69.

Aymonier Étienne, 1900 : Le Cambodge, vol. I, Le royaume actuel, Paris, Ernest Leroux.

BAPTISTE Pierre \& ZÉPHIR Thierry, 2008: L'Art khmer dans les collections du musée Guimet, Paris, Réunion des musées nationaux.

BERNARDINI Pierre, 1975: « Le caodaisme au Cambodge », École pratique des hautes études, $4{ }^{\mathrm{e}}$ section, Sciences historiques et philologiques, Annuaire 1974-1975 p. $1039-1045$

BoISSELIER Jean, 1955 : La statuaire khmère et son évolution, 2 vol., Saigon, EFEO (Publications de l'EFEO, 37).

BoNG Sovath, 2003: «The ceramic chronology of Angkor Borei, Takeo province, Southern Cambodia », Thèse de doctorat (PhD), Mānoa, Department of Anthropology, University of Hawai'i (thèse non publiée).

BouRdEAux Pascal, 2007 : « Approche du bouddhisme Hòa Hảo au Cambodge », Siksā cakr, 8-9, p. 45-54,175-188.

Bourdeaux Pascal, Fischer Christian, Chea Socheat \& PORTE Bertrand, 2013: "Rapport de mission. Recherches sur les roches sculptées du Funan» (rapport non publié).

BouRdonNEAU Éric, 2007 : « Réhabiliter le Funan. Óc Eo ou la première Angkor », BEFEO, 94, p. 111-158.

Bruguier Bruno \& Lacroix Juliette, 2009: Phnom Penh et les provinces méridionales, Guide archéologique du Cambodge, Tome 1, Phnom Penh, Reyum.

CœEDÈs George, 1936: «Études cambodgiennes, XXXI. À propos du Tchen-la d'eau : trois inscriptions de Cochinchine », BEFEO, 36, p. 1-21.

- 1942: Inscriptions du Cambodge, vol. 2, Hanoi, Imprimerie d'Extrême-Orient (Collection de textes et documents sur l'Indochine, 3 ).

Czuma Stanislaw, 1974: «A Masterpiece of Early Cambodian Sculpture », The Bulletin of the Cleveland Museum of Art, 61(4), p. 119-127.

- 1979: «The Case of the Buried Fragments », The Bulletin of the Cleveland Museum of Art, 66(7), p. 289-295.

- 2005: «Krishna Redux. After Centuries of Oblivion, a Great Cambodian Sculpture is Reassemble», The Cleveland Museum of Art Members Magazine, September 2005, p. 12-15.

DAGENS Bruno, 1969: «Étude sur l'iconographie du Bayon (frontons et linteaux) ", Arts Asiatiques, 19, p. 123-167.

Dalsheimer Nadine \& MAnguin Pierre-Yves, 1998 : «Visnu mitrés et réseaux marchands en Asie du Sud-Est: nouvelles données archéologiques sur le Ier millénaire apr. J.-C. », BEFEO, 85, p. 87-123.

DANG Phong, 2010 : Thăng Long-Hà Nội: The Story in a Single Street, introduction d'Andrew Hardy, traduction de Vu Ngoc Quyen, Hanoi, EFEO \& Académi des sciences sociales du Vietnam.

Delaporte Louis, 1880: Voyage au Cambodge. L'architecture Khmer, Paris, Librairie Ch. Delagrave [rééd. Paris, Maisonneuve \& Larose, 1999].
Douglas Janet G., CARò Federico \& FIScher Christian, 2008: «Evidence of sandstone usage for sculpture during the Khmer Empire in Cambodia through petrographic analysis », UDAYA, Journal of Khmer Studies, 9, p. 1-18.

Dupont Pierre, 1955 : La statuaire préangkorienne, Ascona, Artibus Asiae Publishers (Artibus Asia Supplementum, 15).

DUVERnOY Victor, 1924 : Monographie de la province de Longxuyên (Cochinchine), Hanoi, Éditions du moniteur de l'Indochine [2éd. 1930].

FOREST Alain, 1992: Le culte des génies protecteurs au Cambodge, Paris, L'Harmattan.

GiteAu Madeleine, 1965: «Khmer Sculpture and the Angkor Civilization», Londres, Thames \& Hudson.

Goujon-MARINE Euridyce, 2015 : «L'art du Funan : naissance et évolution d'un concept », Mémoire de Master 1, Paris, École du Louvre (mémoire non publié).

Groslier George, 1924: «Promenades artistiques. L'Asram Maha Rosei », Arts et archéologie khmers, tome II, fasc. 1, Paris, Librairie Augustin Challamel, p. $141-146$.

GUY John (dir.), 2014 : Lost Kingdoms. Hindu-Buddhist Sculpture of Early Southeast Asia, New York, The Metropolitan Museum of Art.

JESSUP Helen I. \& ZÉPHIR Thierry (dir.), Angkor et dix siècles d'art khmer, Paris, Réunion des musées nationaux.

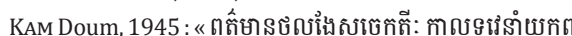

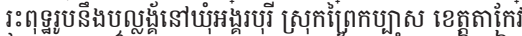

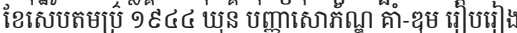
[Rapport sur le transfert de statues de Buddha et d'un piédestal, de la commune d'Angkor Borei, district de Prei Krabas, province de Takeo]», Kampuchea Soriya $17^{\mathrm{e}}$ année, $\mathrm{n}^{\circ} 1$, p. 1-14 ; nº 2, p. 70-93; n 3, p. 137-155

LUNET DE LAJONQUIÈRE Étienne, 1902 : Inventaire descriptif des Monuments du Cambodge, tome I, Paris, Imprimerie nationale \& Ernest Leroux Éditeur (Publications de l'EFEO, 4, Inventaire archéologique de l'Indo-Chine, 1. Monuments du Cambodge)

MALLERET Louis, 1959 : L'archéologie du delta du Mékong tome I, L'exploration archéologique et les fouilles d'OcEo, Paris, EFEO (Publications de l'EFEO, 43).

- 1943 : «Cochinchine, terre inconnue», Bulletin de la Société des études indochinoises, nouvelle série, 19(3), p. 9-28.

MANGUin Pierre-Yves, 2005 : «Nouvelles recherches dans le delta du Mékong», Dossiers d'Archéologie 302 , p. $78-81$

- 2009: «The Archaeology of Funan in the Mekong River Delta: the Oc Eo Culture of Vietnam», in Tingley Nancy (éd.), Arts of Ancient Vietnam: From River Plain to Open Sea, New York \& Houston, Asia Society, Museum of Fine Arts, Yale University Press, p. 100-118.

MARUI Masako, 2002 : « La découverte de statues bouddhiques dans le temple de Banteay Kdei », Aséanie, 10 , p. $65-83$.

MAUgER Henri, 1935 : «Chronique de l'année 1935. Conservation des monuments de CochinchineCambodge », BEFEO, 35(2), p. 488-491.

- 1936a : « L'Àsram Mahà Rosĕi », BEFEO, 36(1), p. 65-95.

- 1936b: «Chronique de l'année 1936. Cochinchine», BEFEO, 36(2), p. 606-607.
- 1937: «Compte-rendu de mes recherches archéologiques au Cambodge. Séance de la $S^{\text {té }}$ Asiatique du 12 juin 1937 », Archives H. Mauger, EFEO (document dactylographié et annoté).

Mus Paul, 1933: "Cultes indiens et indigènes au Champa », BEFEO, 33(1), p. 367-410.

PARMENTIER Henri, 1913 : «Complément à l'Inventaire descriptif des monuments du Cambodge », BEFEO, $13(1)$, p. $1-64$

- 1923 : « Notes d'archéologie indochinoise, II. Vișnu et emplacements de monuments indokhmèrs à Vongthê (Long-xuyèn) », BEFEO, 23(1), p. 275-276.

- 1927 : L'art khmèr primitif, Paris, G. van Oest.

Phon Kaseka, 2004 : «Phnom Borei Survey and Excavation », Académie royale du Cambodge (rapport non publié).

Pomont Jean-Claude, 2015 : Vietnam. L'éphémère et l'insubmersible, Bruxelles, Nevicata (L'âme des peuples).

PoRTE Bertrand, 1996-2016: «Rapports de conservation et restauration de sculptures », Phnom Penh, MNC \& EFEO (rapports non publiés).

- 2002: «La remise au jour du Bouddha de Vat Kompong Luong », Arts Asiatiques, 57, p. 219-222.

- 2006: «La statue du Krș̣na Govardhana du Phnom Da du Musée National de Phnom Penh », UDAYA, Journal of Khmer Studies, 7, p. 199-206.

PORTE Bertrand \& CHEA Socheat, 2008: «Sur les chemins de Tuol Kuhea : note sur un site préangkorien », UDAYA, Journal of Khmer Studies, 9, p.151-168.

SEANG Lin, 2001 : «La légende de Phnom Pros-Phnom Srei », Recueil de contes cambodgiens, vol. V, Phnom Penh, Institut bouddhique à Kompong.

STARK Miriam T., 1998: « The Transition to History in the Mekong Delta: A View from Cambodia», International Journal of Historical Archaeology, 2(3), p. 175-204.

— 1999: avec P. Bion Griffin, Chuch Phoeurn, J. Ledgerwood, M. Dega, C. Mortland, N. Dowling, J. M. Bayman, Bong Sovath, Tea Van, Chhan Chamroeun \& D. K. Latinis, «Results of the 1995-1996 Field Investigations at Angkor Borei, Cambodia », Asian Perspectives, 38(1), p. 7-36.

- 2003a: «Angkor Borei and the Archaeology of Cambodia's Mekong Delta », in Khoo J. (éd.), Art \& Archaeology of Fu Nan: Pre-Khmer Kingdom of the Lower Mekong Valley, Bangkok, Orchid Books, p. 87-106.

- 2003b : « The Chronology, Technology and Contexts of Earthenware Ceramics in Cambodia », in MiksIC J. N. (éd.), Earthenware in Southeast Asia. Proceedings of the Singapore Symposium on Premodern Southeast Asian Earthenwares, Singapour, Singapore University Press, p. 208-229.

- 2003c: avec P. Bishop, D. Penny \& M. Scott, «A 3.5 ka Record of Paleoenvironments and Human Occupation at Angkor Borei, Mekong Delta, Southern Cambodia », Geoarchaeology, 18(3), p. 359-393.

TAYLOR Philip, 2004 : Goddess on the Rise. Pilgrimage and Popular Religion in Vietnam, Honolulu, University of Hawai'i Press.

VINCENT Brice, 2012: «Samrit. Étude de la métallurgie du bronze dans le Cambodge angkorien (fin du $\mathrm{XI}^{\mathrm{e}}$ début du XIII ${ }^{\mathrm{e}}$ siècle », 3 vol., Thèse de doctorat de l'université Paris III - Sorbonne nouvelle (thèse non publiée) 